\title{
Dynamics of star-burst dendrimers in solution in relation to their structural properties
}

\author{
S. Rathgeber ${ }^{\mathrm{a})}$ \\ Forschungszentrum Jülich, Institut für Festkörperforschung-Weiche Materie, D-52425 Jülich, Germany \\ M. Monkenbusch \\ Forschungszentrum Jülich, Institut für Festkörperforschung-Neutronenstreuung, D-52425 Jülich, Germany \\ M. Kreitschmann \\ BASF Schwarzheide GmbH, D-01986 Schwarzheide, Germany \\ V. Urban \\ European Synchrotron Radiation Facility (ESRF), BP 220, F-38043 Grenoble Cedex, France
}

A. Brulet

Laboratoire Léon Brillouin, F-91191 Gif-sur-Yvette Cedex, France

(Received 8 January 2002; accepted 22 May 2002)

\begin{abstract}
We have measured both the static and dynamic structure factors of a single dendrimer with small-angle x-ray scattering (SAXS) and neutron spin-echo spectroscopy under good solvent conditions with the aim of finding a consistent correlation between the structural properties of dendrimers and their dynamic behavior. The samples under investigation were star-burst polyamidoamine dendrimers with generations $g=0$ to 8 in dilute methanol solutions. A model independent approach employing inverse Fourier transformation and square root deconvolution methods has been used to analyze the SAXS data to obtain the pair distance distribution function $p(r)$ and the radial excess electron density profile $\Delta \rho(r)$. In addition, we formulated a model that takes both the colloidal (globular, compact shape with form polydispersity or fuzzy surface) as well as the loose, polymeric (self-avoiding random walk) character of dendrimers into account. With this model we were able to describe the spectra of all dendrimer generations consistently. Parameters discussed as a function of the dendrimer generation are, among others, the correlation length of the density fluctuations (blob radius) $\xi$, the radius of gyration $R_{g}$, the sphere radius $R_{s}$, the form polydispersity $\sigma_{s}$ or analogously, the width of the fuzzy surface region $2 \sigma_{f}$. Both the model-independent approach and the model fits reveal that at least down to the third generation the dendrimers exhibit a rather compact, globular shape. These findings are in agreement with the dynamic results obtained by NSE spectroscopy which probes length scales both larger and much smaller than the dimension of a single dendrimer. The method reveals that the dynamics throughout is dominated by the center-of-mass diffusion-the internal dynamics is suppressed. The diffusion coefficients obtained are close to the values calculated from the Stokes-Einstein relation using the sphere radius $R_{S}$ determined from the SAXS spectra. Dynamically, the dendrimers behave like "hard", solid spheres. (C) 2002 American Institute of Physics. [DOI: 10.1063/1.1493771]
\end{abstract}

\section{INTRODUCTION}

Star-burst dendrimers represent a class of well-defined, highly branched macromolecules. They are built from a central multifunctional core to which multifunctional monomers are added via a protection-deprotection mechanism. This procedure leads to a regular, tree-like structure. The dendrimer structure is defined by the functionality of the core $f$, the functionality of the spacer units $m$, and the number of generations $g$.

The structural properties of dendrimers have been controversial since they were first synthesized by Tomalia et al. ${ }^{1}$ in 1985 . The mass $M$ of a dendrimer grows exponentially as a function of the generation and much faster than the avail-

\footnotetext{
a) Author to whom correspondence should be addressed. Electronic mail: s.rathgeber@fz-juelich.de
}

able volume. This fact raises the question of whether the dendrons, tree-like branches stemming from the central core, are highly stretched to increase the available volume or if there is significant backfolding of the terminal segments to the center of the dendrimer. In the first case one would expect a "hole" in the radial segment density distribution at the dendrimer core, whereas the latter would imply a dense core with a smoothly decreasing segment density with increasing distance from the dendrimer core.

Small-angle scattering experiments with neutrons (SANS) and $\mathrm{x}$ rays (SAXS) are suitable to obtain information on the internal structure and overall shape of dendrimers, such as the fractal dimension $d_{f}$, the distribution of chain ends, and the radial density profile, and are therefore very valuable to validate results obtained from computer simulations. Fractal dimensions of dendrimers have been 
evaluated by Murat and Grest $^{2}$ using molecular dynamics simulations as well as by Mansfield ${ }^{3,4}$ with Monte Carlo methods. Murat and Grest have found $M \propto R_{g}^{d_{f}}$ with $d_{f} \approx 3$ for all generations, with slight deviations occurring only for $g=1$ and $g=2$. A fractal dimension of $d_{f}=3$ indicates that the dendrimers present compact, space-filling structures. On the other hand, a scaling behavior between $d_{f}=2.76$ for $g$ $=9$ down to $d_{f}=2.45$ for $g=6$ obtained by Mansfield would suggest a transition to a looser, more polymeric-like structure with decreasing generation. SANS and SAXS experiments measuring the generation dependence of $R_{g}$ for PAMAM $^{5}$ and poly(propyleneimine) dendrimers ${ }^{6}$ seem to support the $M \sim R_{g}^{3}$ scaling behavior.

Most computer simulations seem to agree that the endgroups of a dendrimer are distributed throughout the molecule $e^{2,3,7-9}$ and are not located predominantly on the outside of the dendrimer, as was postulated by the theory of de Gennes and Hervet. ${ }^{10}$ Topp et al. ${ }^{11}$ however, concluded from SANS experiments on partially labeled dendrimers and the fact that the radius of gyration of the terminal groups $R_{g, T}$ was larger than $R_{g}$ of the entire $g=7$ dendrimer that the endgroups were concentrated near the periphery of the dendrimer. Lyulin et al. ${ }^{12,13}$ later showed that the radius of gyration is not a safe measure of the location of terminal units, since distribution functions with $R_{g, T} \geqslant R_{g}$ can be constructed where most of the endgroups are located within the interior of the dendrimer. In accordance with the concept of backfolding of terminal groups, most simulations support a radial segment density profile having a maximum at the core with a monotonic decrease to the edge, ${ }^{7,8}$ except for a slight local minimum at small radial distances., ${ }^{2,3}$ The notion of a monotonically decreasing density profile for $g=4$ and $g$ $=5$ dendrimers is supported by SANS experiments by Ballauff and co-workers ${ }^{14,15}$ using contrast variation methods. The authors described their SANS spectra by assuming a Gaussian distribution for the segment density profile. Density profiles obtained by IFT and SQDEC-methods from SAXS data by Prosa et al. ${ }^{16}$ decrease monotonically from $g$ $=10$ to $g=7$. For lower generations (from $g=6$ to $g=2$ ) the density profiles display local minima in the periphery which, in the interpretation of the authors, result from a polydispersity of shapes and sizes.

The same authors ${ }^{16}$ also concluded from their SAXS data that a geometry change occurs in dendrimers with decreasing generation number. A gradual transition in the $q$ dependence of the data at high scattering vectors, from a $q^{-4}$ power-law behavior for $g=10$, which would be typical for a dense spherical object with smooth surface, to a $\mathrm{q}^{-5 / 3}$ powerlaw behavior for $g=3$, which would be characteristic for a loose polymeric structure under good solvent condition, was taken as the basis for this conclusion. Accordingly, the form factors lose more features in the high-q region, e.g., higher order maxima. The authors claimed that their observations were in qualitative agreement with simulation results from Mansfield, ${ }^{3,4,17}$ who, however, observed a $q^{-1 / 2}$ behavior $(\theta-$ condition) at high $q$ values also for high-generation dendrimers.

In our opinion, the previously reported work is not totally consistent and requires a more detailed analysis. Be- cause the comprehensive understanding of the static properties is essential for the interpretation of the dendrimer dynamics, we will present in Sec. IV A a detailed discussion of our static measurements and results.

Our work on the dynamic properties of dendrimers was motivated by the extensive studies of Richter et al. ${ }^{18-20}$ on the dynamics of star polymers, which represent another class of highly branched macromolecules. The authors showed that for these systems the internal dynamics reflects the star polymer architecture. Recent theoretical work indicates that this should also be true for dendritic polymers. ${ }^{21,22}$ The aim of this work is to find a consistent correlation between the structure of the dendrimers and their dynamic behavior.

Richter and co-workers found that low-functionality stars show the same behavior as linear homopolymers. A simple transition from diffusional dynamics at low $q$ values to Zimm dynamics at high $q$ values is observed. In contrast, for high-functionality stars, the authors found a pronounced minimum in the reduced relaxation rates $\Omega / q^{3}$ on the same length scales $2 \pi / q^{\star}$, where the structure factor exhibits a maximum in Kratky representation. Since the reduced linewidth scales with the scaling variable $q R_{g, a}\left(R_{g, a}=\right.$ radius of gyration of one arm) in the same way as the form factor, Richter et al. draw an analogy to the phenomenon of de Gennes narrowing in the case of liquids, where the structure factor $S(q)$ renormalizes the intrinsic relaxation rate of the density fluctuations $\Omega$ to an effective rate $\Omega_{\text {eff }}=\Omega / S(q)$. Whereas de Gennes narrowing denotes a collective phenomenon involving different particles, the effect described above occurs for the density fluctuations within one entity, however, containing a large number of monomeric segments.

For dendrimers of various branching topology, local stiffness, and different generations, La Ferla ${ }^{21}$ and Ganazzoli et $a l .{ }^{22}$ calculated the dynamic structure factor in the framework of the Rouse-Zimm theory. Compared to star polymers, the model predicts an even deeper local minimum located at $q R_{g} \approx 2.08$ for the reduced relaxation rate, except for dendrimers with high local stiffness. In contrast to the findings for star polymers, the depth and the width of the minimum decreases with increasing stiffness. High local stiffness would prevent a space-filling arrangement in the dendrimer interior and thus reduce the screening of the hydrodynamic interaction.

This paper is outlined as follows: In Sec. II A we give a short theoretical introduction to the first cumulant evaluation and summarize the most important equations necessary for the evaluation of the dynamic NSE data. In Secs. II B 1 and II B 2 we would like to give a short introduction to the inverse Fourier transformation (IFT) and square root deconvolution (SQDEC) methods developed by Glatter. ${ }^{23}$ A comprehensive introduction to the model we formulated for the analysis of the static SAXS data is given in Sec. II C. Section III describes the samples and summarizes the experimental conditions for the SAXS and NSE experiments. In Sec. IV A the static SAXS results are presented and discussed with the emphasis on putting the structural characteristics of the dendrimers in relation to those of low- and high-functionality stars. In Sec. IV B the dynamic NSE results are shown and the relation between the structural and dynamical properties 
of the dendrimers and the differences from previous results on high-functionality stars are discussed. The major conclusions are summarized in Sec. V.

\section{THEORY}

\section{A. NSE-First cumulant evaluation}

Neutron spin-echo spectroscopy measures the coherent intermediate structure factor $S(q, t)$ on length scales where we are able to resolve not only the diffusion of the overall dendrimer but also internal relaxation processes. Since explicit theoretical predictions for the dynamic structure factor of a dendrimer in dilute solution are still missing, we discuss our data using a first cumulant approach, which is discussed in more detail in Ref. 24. In the framework of linear response theory, the time development of the density vector $\rho(q, t)$ satisfies the condition

$$
\frac{\partial \varrho(q, t)}{\partial t}=-\underline{\mathcal{L}} \varrho(q, t),
$$

where $\mathcal{L}$ is a real, linear, and time-independent operator acting on the position vectors of all segments in the solution. From this general equation, via a Mori-Zwanzig projection formalism, a generalized Langevin equation can be derived, i.e.,

$$
\begin{aligned}
\frac{\partial \varrho(q, t)}{\partial t}= & -\underline{\Omega}(q) \varrho(q, t)+\int_{0}^{t} d u \underline{\underline{\Phi}}(q, t-u) \varrho(q, u) \\
& +f(t),
\end{aligned}
$$

where $f(t)$ denotes the random Langevin forces acting from the surrounding medium, which is considered as a heat bath acting on each segment. The memory matrix $\Phi(q, t)$ takes the viscoelastic properties of the medium into account, and the so-called relaxation frequency or mobility matrix $\Omega(q)$ describes the (short-time) elastic response. Multiplying Eq. (2) from the right with $\varrho^{\star}(q, 0)$ and taking equilibrium averages finally leads to the equation for the dynamic structure factor $\underset{\underline{S}}{S}(q, t)=\left\langle\varrho(q, t) \varrho^{\star}(q, 0)\right\rangle$

$$
\frac{\partial \underline{\underline{S}}(q, t)}{\partial t}=-\underline{\underline{\Omega}}(q) \underline{\underline{S}}(q, t)+\int_{0}^{t} d u \Phi(q, t-u) \underline{\underline{S}}(q, u) .
$$

For short times memory effects (second term) can be disregarded, leading to a decoupled linear differential equation system for $\underset{S}{S}(q, t)$, that can easily be solved. For a onecomponent system, such as a fully protonated dendrimer in deuterated solvent (the solvent can be eliminated by the incompressibility condition), the short-time decay of the NSE signal should follow a simple single-exponential decay

$$
\begin{aligned}
\frac{d S(q, t)}{d t} & =-\Omega(q) S(q, t) \\
& \Rightarrow S(q, t)=S(q, t=0) \exp [-\Omega(q) t] .
\end{aligned}
$$

Only the static properties of the system enter the calculation of the first cumulant

$$
\Omega(q)=\frac{k_{B} T q^{2} \mu(q)}{S(q, 0)},
$$

with $\mu(q)=\frac{\left\langle\varrho(q, 0) \underline{\left.\underline{\mathcal{L}} \varrho^{\star}(q, 0)\right\rangle}\right.}{k_{B} T q^{2}}$,

where $\mu(q)$ is the so-called generalized mobility. In the framework of the random phase approximation (RPA) $\mu(q)$ or $\Omega(q)$ can be determined, but assumptions about the detailed interaction between segments must be made. ${ }^{24}$

The Zimm model takes hydrodynamic interaction between the segments via the flow field of the surrounding solvent into account. The generalized mobility can be written as

$$
\begin{gathered}
\mu(q)=\frac{\sum_{l, m}^{N}\left\langle q \underline{\underline{D}}_{l m} q \exp \left(-i q \underline{r}_{l m}\right)\right\rangle}{k_{B} T q^{2}}, \\
\text { with } \quad \underline{D}_{l m}=\frac{k_{B} T}{\zeta} \delta_{l m}+\left(1-\delta_{l m}\right) \underline{\underline{T}}_{l m},
\end{gathered}
$$

where $N$ denotes the number of segments and $\zeta$ is the local friction coefficient. The Oseen tensor $\underline{\underline{T}}_{l m}$ describes a flow field, the strength of which decays slowly with the inverse of the distance between two segments, leading to a strong coupling of the dynamics of different segments. It was shown by Burchard et al. ${ }^{25}$ that for Zimm dynamics the generalized mobility can be expressed to high accuracy by the sum of a hydrodynamically preaveraged term $\mu^{\mathrm{pre}}(q) \propto \Sigma_{l, m}^{N}\left\langle q D_{l m} q\right\rangle$ $\times\left\langle\exp \left(-i q \underline{r}_{l m}\right)\right\rangle$ and a correction term $\Delta \mu(q)$

$$
\begin{aligned}
\mu^{\text {pre }}(q)= & \frac{1}{3 \pi^{2} \eta_{s}} \int_{0}^{\infty} S\left(\sqrt{q^{2}+u^{2}}\right) d u, \\
\Delta \mu(q)= & \frac{q^{2}}{30 \pi^{2} \eta_{s}} \int_{0}^{\infty} u^{-2}\left[S\left(\sqrt{0.72 q^{2}}\right)\right. \\
& \left.-S\left(\sqrt{0.72 q^{2}+u^{2}}\right)\right] d u,
\end{aligned}
$$

where $\eta_{s}$ is the solvent viscosity.

In case of noninteracting segments undergoing Rouse relaxation, according to Eq. (6) we obtain $D_{l m}=\left(k_{B} T / \zeta\right) \delta_{l m}$ and the mobility is then simply given by $\mu=N / \zeta$. As a consequence of the local character of the friction, the mobility does not depend on $q$. For the first cumulant $\Omega_{R}$ we derive with Eq. (5) and the limits of the static structure factor (1) $S(q) \approx N^{2}$ for $q \ll R_{g}^{-1}$ and $(2) S(q) \approx 12 N(q l)^{-2}$ for $l^{-1}$ $\gg q \gg R_{g}^{-1}$

$$
\begin{aligned}
& \text { (1) } \Omega_{R}(q)=q^{2} D_{R}=q^{2} \frac{k_{B} T}{N \zeta} \text { for } q \ll R_{g}^{-1}, \\
& \text { (2) } \Omega_{R}(q)=\frac{k_{B} T}{12} \frac{l^{2}}{\zeta} q^{4} \quad \text { for } l^{-1} \gg q \gg R_{g}^{-1},
\end{aligned}
$$

with $l$ being the segment length and $D_{R}$ the Rouse center-ofmass diffusion coefficient. The relaxation rate increases with $q^{4}$ for segmental relaxation and with $q^{2}$ for simple center-ofmass diffusion.

As a result of the long-range interaction in the case of Zimm dynamics, the mobility is now a function of the scattering vector. The high- $q$ limits for the static structure factor are given by (a) $S(q) \approx 12 N(q l)^{-2}$ for the $\theta$-condition and 
(b) $S(q) \propto(q l)^{-1 / \nu}$ with the Flory-Huggins parameter $\nu$ $=3 / 5$ for good solvent conditions. Insertion into Eq. (7) leads to

(a) $\mu_{Z \theta}^{\mathrm{pre}}(q)=\frac{2}{\pi} \frac{N}{\eta_{s} l^{2}} q^{-1}$ for $\theta$-solvent, and

(b) $\mu_{Z g}^{\mathrm{pre}}(q) \propto \frac{1}{\eta_{s} l^{5 / 3}} q^{-2 / 3}$ for good solvent

for the $q$-dependent mobilities. The corresponding first cumulants $\Omega_{Z}$ now read

(a) $\Omega_{Z \theta}(q)=\frac{1}{6 \pi} \frac{k_{B} T}{\eta_{s}} q^{3}$ for $\theta$-solvent, and

(b) $\Omega_{Z g}(q)=0.071 \frac{k_{B} T}{\eta_{s}} q^{3}$ for good solvent.

The relaxation rates increase with $q^{3}$ for segmental relaxation. Not preaveraging the Oseen tensor just leads to a renormalization of the prefactors from $1 /(6 \pi) \approx 0.053$ to 0.063 and from 0.071 to 0.079 for $\theta$-and good solvent conditions, respectively. ${ }^{26,27}$ Independent of the solvent quality, the $q^{3}$ dependence is preserved.

For the Zimm center-of-mass diffusion coefficients $D_{Z}$, the following expressions
(a) $D_{Z \theta}=0.192 \frac{k_{B} T}{\eta_{s} R_{E}}$ for $\theta$-solvent, and
(b) $D_{Z g}=0.203 \frac{k_{B} T}{\eta_{s} R_{E}}$ for good solvent

can be derived, where $R_{E}$ is the end-to-end distance. ${ }^{28}$

Practical fitting of the NSE spectra involves $\Omega$ as the only parameter. By fitting the data for each $q$ value separately, the relaxation rates or relaxation times $\left(\tau=\Omega^{-1}\right)$ can be determined as a function of the scattering vector or equivalent as a function of the length scale $r=2 \pi / q$.

\section{B. SAXS-Model independent approach}

In this paper we analyze our small-angle x-ray (SAXS) data via a model-independent approach as well as by direct modeling. The latter will be presented in Sec. IIC. The model-independent evaluation involves an indirect Fourier transformation (IFT), which provides the pair distance distribution function $p(r)$, or, equivalently the correlation function $\gamma(r)=r^{1-\operatorname{dim}} p(r)$, where dim defines the type of symmetry $(\operatorname{dim}=1$ lamellar, $\operatorname{dim}=2$ cylindrical, and $\operatorname{dim}=3$ spherical). Assuming spherical symmetry of the scattering objects in a subsequent step via a square-root deconvolution (SQDEC) of $p(r)$, the radial excess electron density distribution $\Delta \rho(r)$ can be derived.

\section{Inverse Fourier transformation (IFT)}

The intensity $I_{\exp }(q)^{54}$ measured in a SANS or SAXS experiment is given by the Fourier transform of the pair distance distribution function $p(r)$

$$
I_{\exp }(q)=4 \pi \int_{0}^{\infty} p(r) \frac{\sin (q r)}{q r} d r .
$$

Due to the finite experimental range of scattering vectors, the potentially significant influence of instrumental smearing effects, and insufficiently corrected background scattering, it is not possible to derive $p(r)$ by direct inverse Fourier transformation. We therefore applied an IFT method first introduced by Glatter. ${ }^{23}$ The theoretical aspects of this method and its experimental applications are discussed elsewhere. ${ }^{23,29-31}$ From the approximated pair distance distribution function $p_{\mathrm{IFT}}(r)$, the radius of gyration

$$
R_{g}^{2}=\frac{\int_{0}^{\infty} p_{\mathrm{IFT}}(r) r^{2} d r}{2 \int_{0}^{\infty} p_{\mathrm{IFT}}(r) d r},
$$

and the forward scattering intensity

$$
I_{\mathrm{IFT}}(q=0)=4 \pi \int_{0}^{\infty} p_{\mathrm{IFT}}(r) d r,
$$

can be calculated. In particular, we used the IFT program of Pedersen for the calculation of $p_{\mathrm{IFT}}(r)$ from our SAXS data. $^{30}$

From the sampling theorem, ${ }^{32}$ predictions can be made about the minimal and maximal length scale, $\Delta r_{\mathrm{IFT}}$ and $D_{\text {max }}$, that can be resolved in real space by indirect Fourier transformation of a small-angle scattering data set. For a set of $P$ data points at scattering vectors with values between $q_{\text {min }} \leqslant q \leqslant q_{\text {max }}$ and a step width of $\Delta q$, we derive the conditions

$$
\begin{aligned}
& D_{\max } \leqslant \frac{\pi}{\Delta q} \quad \text { or } \quad D_{\max } \leqslant \frac{\pi}{q_{\min }}, \\
& \Delta r_{\mathrm{IFT}}=\frac{\pi}{P \Delta q} \quad \text { or } \quad \Delta r_{\mathrm{IFT}}=\frac{\pi}{q_{\max }} .
\end{aligned}
$$

In our SAXS experiment not $\Delta q=5.5 \times 10^{-3} \mathrm{~nm}^{-1}$ but $q_{\text {min }}=0.08 \mathrm{~nm}^{-1}$ and $q_{\max }=3.5 \mathrm{~nm}^{-1}$ (see Sec. III B) are the limiting factors leading to an upper and lower resolution limit of $D_{\max }=39 \mathrm{~nm}$ and $\Delta r_{\mathrm{IFT}}=0.9 \mathrm{~nm}$, respectively. For the calculation of $p(r)$ the program routine requires an estimation of the maximum particle dimension $D$. Müller and Glatter $^{31}$ have shown that an overestimation of the true particle dimension does not lead to significant changes in $p(r)$. We obtained stable results by setting $D$ up to $4 \times R_{g}$, which gives $D \approx 16 \mathrm{~nm}$ for the $g=8$ PAMAM dendrimer. This value clearly lies below the theoretical upper resolution limit $D_{\text {max }}$ of the IFT method. The value for $\Delta r_{\text {IFT }}$ obtained from Eq. (17) underestimates the lower resolution limit. Extensive tests indicate that the resolution is better estimated by the relation $\Delta r_{\mathrm{IFT}} \approx 1 / q_{\max }$, which would result in a resolution of about $\approx 0.3 \mathrm{~nm}$ for our particular case. ${ }^{30}$

\section{Square-root deconvolution method (SQDEC)}

The radial excess scattering length or electron density distribution $\Delta \rho(r)$ (scattering length or electron density of the polymer with respect to the solvent) is related to the pair distance distribution function by

$$
p(r)=r^{2} \int_{-\infty}^{\infty} \Delta \rho(x) \Delta \rho(x-r) d x,
$$


where $r$ is the distance from the center of spherical symmetry $(\operatorname{dim}=3)$. In practice, statistical and systematic errors, etc. will make a direct deconvolution impossible. $\Delta \rho_{\mathrm{SQDEC}}(r)$ can be obtained from $p_{\mathrm{IFT}}(r)$ via an (indirect) square-root deconvolution routine introduced by Glatter. ${ }^{33}$ This procedure is similar to the IFT method, except that the resulting equations are nonlinear and have to be solved iteratively.

In particular, we used the SQDEC program of Pedersen for the deduction of $\Delta \rho_{\mathrm{SQDEC}}(r)$ from $p_{\mathrm{IFT}}(r) .^{30}$ The program follows the procedures described below and is discussed more extensively in Refs. 29-31 and 33-35. The density distribution is approximated by a series of $M$ equidistant box functions $B_{m}^{0}(r)$ (zeroth-order spline functions)

$$
\begin{aligned}
\Delta \rho_{\mathrm{SQDEC}}(r) & =\sum_{m=1}^{M} b_{m} B_{m}^{0}(r) \text { for } 0 \leqslant r \leqslant R_{\max } \\
& =0 \quad \text { for } r>R_{\max },
\end{aligned}
$$

where $b_{m}$ denotes the constant excess electron (scattering length) density in each shell and $R_{\max }$ is an estimate for the maximal particle radius. Insertion into Eq. (18) leads to

$$
\begin{aligned}
& p_{\mathrm{SQDEC}}=\sum_{m=1}^{M} V_{m m}(r) b_{m}^{2}+2 \sum_{m>n} V_{m n}(r) b_{m} b_{n}, \\
& \text { since } V_{n m}(r)=V_{m n}(r) .
\end{aligned}
$$

The overlap integrals $V_{n m}(r)$ that give the overlap of the $m$ th and $n$th box function as a function of a displacement $r$ also contain the symmetry-dependent factors $r^{\text {dim-1 }}$. The SQDEC method applied here assumes spherical symmetry, in which case analytical expressions can be obtained for $V_{n m}(r)$. It has been shown by Glatter ${ }^{33,35}$ that the SQDEC gives reasonable results even in the case of small deviations from spherical symmetry. The nonlinear set of equations for $b_{m}$ is defined by the optimizing condition $\left(\chi^{2}+\lambda N_{c}\right)=\min$ with

$$
\chi^{2}=\sum_{i=1}^{P^{\prime}} \frac{\left[p_{\mathrm{SQDEC}}\left(r_{i}\right)-p_{\mathrm{IFT}}\left(r_{i}\right)\right]^{2}}{\sigma_{i}^{2}},
$$

and the constraint

$$
N_{c}=\sum_{m=1}^{M-1}\left[b_{m+1}-b_{m}\right]^{2},
$$

where $P^{\prime}$ is the number of discrete data points of $p_{I F T}\left(r_{i}\right)$ each having a standard error $\sigma_{i}$. The optimal value of the Lagrangian multiplier $\lambda$ is determined by a discrepancy criterion. $^{30}$

We obtain $\Delta \rho(r)$ in absolute units with the condition

$$
\sum_{m=1}^{M} b_{m} V_{m}=\frac{V_{W}}{N_{A}} \Delta \rho,
$$

where the $V_{m}$ are volumes of spherical shells of thickness $\Delta r_{\mathrm{SQDEC}}=R_{\max } / M$ determined by the number of box functions $M$ used in the deconvolution procedure. The sum over all products $V_{m} b_{m}$ should be equal to the product of the polymer volume $V_{W}$ determined from the forward scattering intensity [Eq. (15)] and the theoretical excess electron (scat-

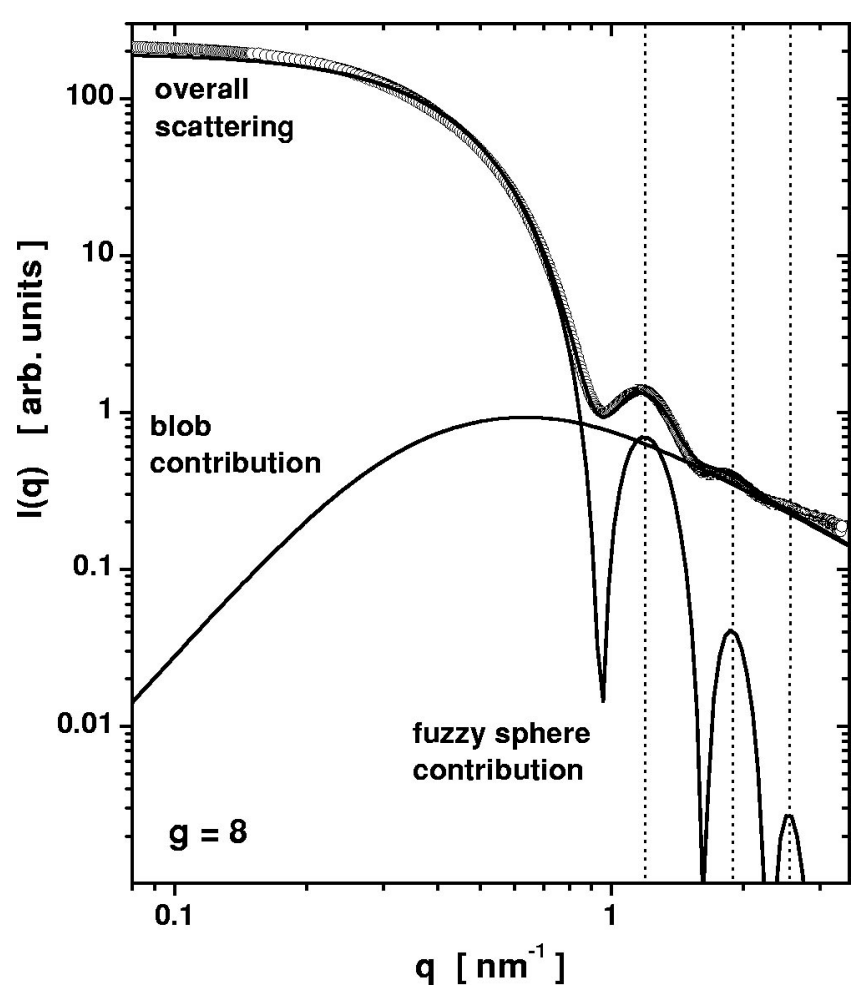

FIG. 1. Comparison between the theoretical predictions calculated from the fuzzy sphere model with internal density fluctuations and the experimental SAXS spectrum (circles) obtained for the $g=8$ PAMAM dendrimer. The separate contribution of the blob scattering and the contribution of the overall dendrimer shape (fuzzy sphere) to the total scattering are also shown.

tering length) density $\Delta \rho$. The shell thickness $\Delta r_{\mathrm{SQDEC}}$ determines the resolution of the method in real space. The maximal number of box functions used in the program of Pedersen is $M=40$. With the estimated maximal radius $R_{\max }$ (required as an input parameter) set to $1.5 \times R_{g}$, this would lead to a spatial resolution of $\Delta r_{\mathrm{SQDEC}}=0.15 \mathrm{~nm}$ for the $g$ $=8$ dendrimer, a value smaller than the resolution of the IFT method. In practice, we used $M \leqslant 20$ box functions so that $\Delta r_{\mathrm{SQDEC}} \approx \Delta r_{\mathrm{IFT}} \approx 0.3 \mathrm{~nm}$ was always satisfied for all dendrimer generations.

\section{SAXS-Data modeling}

\section{Sphere with fuzzy shell and internal density fluctuations}

In the dendrimer scattering spectra, as for example plotted in Fig. 1 for the $g=8$ dendrimer, two different regimes can be clearly distinguished. At low $q$ values the scattering appears to show the typical behavior expected for spherical objects with the occurrence of higher order maxima for the dendrimers with generation $g \geqslant 6$. In the high- $q$ regime the dendrimer form factor $P(q)$ is dominated by a power-law dependence originating from the density fluctuations on length scales smaller than the dimension of the dendrimer. The scattering on length scales smaller than the correlation length $\xi$ of the density fluctuations is analogous to that of a semidilute polymer solution and the exponent of the powerlaw dependence can be derived from the Flory-Huggins parameter $\nu$. Within a so-called "blob", a spherical volume 
with radius $\xi$, the dendrons have to be described as a selfavoiding random walk and excluded-volume interaction has to be considered. ${ }^{36}$

Following a similar ansatz of Dozier et al. ${ }^{37}$ for highfunctionality stars, which has also, in a different modification, been successfully applied to polymeric micelles, ${ }^{38}$ we split the dendrimer form factor into the sum of two terms

$$
P(q)=P_{\text {shape }}(q)+a_{b}^{\prime} 4 \pi \int_{0}^{\bar{\xi}} r^{2} \gamma(r) \frac{\sin (q r)}{q r} d r,
$$

where $a_{b}^{\prime}$ denotes the relative amplitude of the blob scattering contribution. The first term $P_{\text {shape }}(q)$ denotes the form factor most appropriate to describe the overall shape of the dendrimer (see below) and the second term $P_{\text {blob }}(q)$ represents the Fourier transform of the density correlation function $\gamma(r)$ describing the density fluctuations on length scales smaller than the blob radius. In the Daoud-Cotton model $\xi$ $=\xi(r)$ is expected to increase with increasing radial distance from the center. ${ }^{36}$ However, in the $q$ range of interest only the largest, outermost blobs should give a significant contribution to the scattering signal. Therefore, we neglect the $r$ dependence of the blob radius and replace $\xi(r)$ by an effective blob radius $\bar{\xi}$. Since the correlation function of the density fluctuations is equal to zero for length scales larger than the correlation length $\bar{\xi}$, the integration in Eq. (23) is limited to $r \leqslant \bar{\xi}$. Taking the sum of both terms instead of their convolution is strictly valid only if the $q$ ranges of both scattering contributions are clearly separated, allowing us to neglect interference terms. As a result of the density fluctuations, the center of mass of the dendrimer itself undergoes random displacements (Brownian diffusion) around a fixed origin. Following the model introduced by Beaucage, ${ }^{39}$ this can be taken into account by substituting the scattering vector $q$ by the reduced parameter $q^{\star}$

$$
q^{\star}=\frac{q}{\left[\operatorname{erf}\left(q R_{g} / \sqrt{6}\right)\right]^{3}},
$$

where erf denotes the error function. In this way a "cutoff" of the power-law contribution to low $q$ values, where the shape contribution should be dominant, is ensured (see Fig. 1). According to the results of Daoud and Cotton ${ }^{36}$ for $r$ $\leqslant \bar{\xi}$, the density correlation function $\gamma(r)$ is set to

$$
\gamma(r) \propto r^{\mu-2}, \quad \text { with } \mu=\nu^{-1}-1,
$$

where $\nu$ is the Flory-Huggins parameter, which equals $3 / 5$ for good solvent conditions, $1 / 2$ for $\theta$-solvents and 2/3 in the case that the dendrons are stretched. An analytical expression for the blob scattering can be obtained by inserting a "cutoff" factor $\exp (-r / \bar{\xi})$ into the integral of Eq. (23) and extending the integration to infinity. The blob scattering contribution to the dendrimer form factor then finally reads

$$
P_{\mathrm{blob}}(q)=\frac{a_{b}}{\mu q_{b}^{\star}} \frac{\sin \left[\mu \tan ^{-1}\left(q_{b}^{\star}\right)\right]}{\left[1+q_{b}^{\star 2}\right]^{\mu / 2}},
$$

with $q_{b}^{\star}=q^{\star} \bar{\xi}$ and $a_{b}=a_{b}^{\prime} \cdot 4 \pi \mu \bar{\xi}^{\mu+1} \Gamma(\mu)$, so that $P_{\text {blob }}(q$ $\rightarrow 0) / a_{b}=1$. $\Gamma(\mu)$ denotes the gamma function.
The relative amplitude $a_{b}$ of the blob scattering to the amplitude of the contribution stemming from the overall shape can be considered in analogy to the star macromolecules. ${ }^{40,41}$ Since there is no correlation between segments belonging to different blobs, the scattering intensity $I(q=1 / \bar{\xi})$ on length scales of the effective blob radius arises from the incoherent superposition of the coherent scattering from within the blobs: $I(q=1 / \bar{\xi}) \approx n_{b} N_{b}^{2}$, where $n_{b}$ denotes the number of blobs in the dendrimer and $N_{b}$ is the number of segments in one blob. In the limit $q=0$ all contributions add coherently, resulting in a total intensity of $I(q=0) \approx\left(n_{b} N_{b}\right)^{2}=N_{t}^{2}$, with $N_{t}$ being the overall number of segments in the dendrimer. The amplitude $a_{b}$ $=I(1 / \bar{\xi}) / I(0)$ is simply given by the ratio of the number of scatterers in one blob $N_{b}$ to the total dendrimer segment number $N_{t}$

$$
\begin{aligned}
& a_{b}=\frac{n_{b} N_{b}^{2}}{N_{t}^{2}}=\frac{N_{b}}{N_{t}}, \\
& \text { with } \quad N_{t}=\frac{f}{m-1}\left(m^{g+1}-1\right)+1=2^{g+3}-3,
\end{aligned}
$$

where $m=2$ is the functionality of the branching unit, $f=4$ is the functionality of the core, and $g$ is the generation of the dendrimer.

From the reduced slope in the intermediate $q$ region of the dendrimer form factor (see Sec. IV A) we can predict that the dendrimers are not perfectly spherical in shape. In principle, we can imagine three different scenarios: (1) the dendrimer morphology is ellipsoidal rather than spherical, as seen in some computer simulation studies; ${ }^{3,42}$ (2) the dendrimers are homogeneous spheres with a sharp edge but with a certain (Gaussian) polydispersity in the sphere radii and; last but not least (3) their density profile is constant in the dense, homogeneous core region but decays smoothly (monotonically) to zero in a softer shell region (fuzzy edge).

For a rotational ellipsoid with main axes $R_{\mathrm{el}}, R_{\mathrm{el}}$ and $\epsilon R_{\mathrm{el}}$ in Eq. (23), $P_{\text {shape }}(q)$ has to be replaced by ${ }^{43}$

$$
\begin{aligned}
& P_{\text {shape }}^{\mathrm{el}}\left(q, \epsilon, R_{\mathrm{el}}\right)=\int_{0}^{\pi / 2} P_{s}\left(q, r^{\prime}\right) \sin \alpha d \alpha, \\
& \text { with } \quad r^{\prime}=R_{\mathrm{el}} \sqrt{\sin ^{2} \alpha+\epsilon^{2} \cos ^{2} \alpha},
\end{aligned}
$$

and

$$
\begin{aligned}
P_{s}\left(q, r^{\prime}\right) & =A_{s}\left(q, r^{\prime}\right)^{2} \\
& =\frac{9}{\left(q r^{\prime}\right)^{6}}\left[\sin \left(q r^{\prime}\right)-q r^{\prime} \cos \left(q r^{\prime}\right)\right]^{2} .
\end{aligned}
$$

For a sphere with radius $R_{S}$ and Gaussian form polydispersity with standard deviation $\sigma_{s}$, the normalized form factor reads 


$$
\begin{aligned}
P_{\text {shape }}^{s}\left(q, R_{s}, \sigma_{s}\right)= & \sqrt{\frac{2}{\pi}} \frac{1}{\sigma_{s}}\left[1+\operatorname{erf}\left(\frac{R_{s}}{\sqrt{2} \sigma_{s}}\right)\right]^{-1} \\
& \times \int_{0}^{\infty} P_{s}(q, r) \exp \left[-\frac{\left(r-R_{s}\right)^{2}}{2 \sigma_{s}^{2}}\right] d r .
\end{aligned}
$$

The integrations have been performed numerically.

Another model already successfully applied to polymer micelles describes the fuzzy, nonideal surface of a spherical object by a convolution of a density profile of a homogeneous sphere with a Gaussian $\exp \left(-r^{2} / \sigma_{f}^{2}\right)$. Since the Fourier transform of a convolution product simply splits into the product of the Fourier transform of the multipliers, we obtain a simple analytical expression for the normalized scattering amplitude

$$
\begin{aligned}
A_{f s}\left(q, R_{f}, \sigma_{f}\right) & =\int_{0}^{\infty} \Delta \rho(r) r^{2} \frac{\sin (q r)}{q r} d r / \int_{0}^{\infty} \Delta \rho(r) r^{2} d r \\
& =A_{s}\left(q, R_{f}\right) \exp \left(-\frac{q^{2} \sigma_{f}^{2}}{4}\right),
\end{aligned}
$$

and for the form factor $P_{\text {shape }}^{f s}\left(q, R_{f}, \sigma_{f}\right)=A_{f s}\left(q, R_{f}, \sigma_{f}\right)^{2}$. Here, $2 \sigma_{f}$ is a measure for the width of the soft shell region and $R_{f}$ is the radius of an analogous homogeneous sphere with the same mass and core density (see the insert of Fig. 5). The pair distance distribution function $p(r)$ and the segment density profile $\Delta \rho(r)$ were obtained by numerical inverse Fourier transformation of the form factor $P_{f s}(q)$ and of the amplitude $A_{f s}(q)$, respectively [see Eq. (31) and (13)].

In summary, we describe our static SAXS data with a form factor which is given by a sum of two contributions

(i) A term that takes the scattering stemming from the internal density fluctuations into account. The effective correlation length (blob radius) $\bar{\xi}$ of these fluctuations and the amplitude $a_{b}$ of the blob scattering relative to the shape contribution are the adjustable parameters.

(ii) The second term describes the overall shape of the dendrimer. Here, we distinguish three cases.

(a) Polydisperse spheres with the sphere radius $R_{s}$ and the standard deviation $\sigma_{s}$ of the Gaussian form polydispersity as free parameters.

(b) Rotational ellipsoids with the main axes $R_{\mathrm{el}}, R_{e l}$ and $\epsilon R_{\mathrm{el}}$. The fit parameters are the radius of gyration $R_{g}=R_{\mathrm{el}} \sqrt{\left(2+\epsilon^{2}\right) / 5}$ and the aspect ratio $\epsilon$.

(c) The fuzzy edge model with the width $2 \sigma_{f}$ of the surface region and the radius $R_{f}$ of the analogous, homogeneous sphere with a sharp edge, the same mass and same density in the core region as adjustable parameters.

\section{Dendrimer and star form factor}

To put our results into the context of previous studies performed on high-functionality stars, we also compared our data to the theoretical form factor obtained for dendrimers from which the special case of a star polymer $(g=1)$ can be derived. Using the cascade theory, Burchard et al. ${ }^{25}$ were the first to derive an expression for the form factor of star-like Gaussian dendrimers of special architecture $(f=3, m=2)$ but arbitrary number of segments $n$ with length $l$ between the branching units. Hammouda ${ }^{44}$ derived a generalized analytical expression for the dendrimer form factor with an arbitrary functionality of the core and the branching units.

The dendrimer form factor can be expressed in terms of the dimensionless scattering variable $\alpha=q^{2} l^{2} / 6$, the scattering amplitude $A(\alpha, n)$, and the form factor $P(\alpha, n)$ of the linear chains between the branching points. In the following we also would like to take the case into account where the dendrimer core is a linear chain built by $n^{\prime}$ segments of length $l^{\prime}$, with $m^{\prime}$ being the functionality of the end segments. The different cross- and self-correlation terms as defined by Hammouda ${ }^{44}$ then read

$$
\begin{aligned}
P_{s b}^{s}(q) & =\frac{m^{g}-1}{m-1} P(\alpha, n)+\frac{1}{f} P\left(\alpha^{\prime}, n^{\prime}\right), \\
P_{s b}^{f}(q)= & 2 A(\alpha, n)^{2} \sum_{k=1}^{g} m^{k-1} \sum_{i=k+1}^{g} m^{i-k} \\
& \times \exp [-\alpha n(i-k-1)]+2 A(\alpha, n) A\left(\alpha^{\prime}, n^{\prime}\right) \\
& \times \sum_{j=1}^{g} m^{j-1} \exp [-\alpha n(j-1)] \\
P_{i b}(q)= & A(\alpha, n)^{2} \sum_{k=1}^{g} m^{k-1} \sum_{i=1}^{g} m^{i-1} \\
& \times \exp \left[-\left(\alpha n(i+k-2)+\alpha^{\prime} x\right)\right] \\
P_{s b}^{a}(q)= & A(\alpha, n)^{2}(m-1) \sum_{k=2}^{g} m^{k-1} \sum_{i=1}^{k} \sum_{j=1}^{g-i} m^{j-1} \\
& \times \exp [-\alpha n(k-i-1+j)],
\end{aligned}
$$

where $\alpha^{\prime}=q^{2} l^{\prime 2} / 6$. The dendrimer form factor normalized to unity is given by

$$
\begin{aligned}
P(q)= & {\left[f\left(P_{s b}^{s}(q)+P_{s b}^{f}(q)+P_{s b}^{a}\right)+2 m^{\prime 2} P_{i b}\left(q, x=n^{\prime}\right)\right.} \\
& \left.+2 m^{\prime}\left(m^{\prime}-1\right) P_{i b}(q, x=0)\right] / N_{t}^{2},
\end{aligned}
$$

with $N_{t}$ being the total number of segments in the dendrimer as defined in Eq. (27). To mimic excluded volume interaction, following procedures previously described for star polymers, ${ }^{19}$ we redefine $\alpha_{\nu}=6 \alpha /[(2 \nu+1)(2 \nu+2)]$ and replace $(n g)$ by $(n g)^{2 \nu}$. In addition, for the scattering amplitude and the form factor of the linear units, Eqs. (34) and (35) must be inserted instead of their Gaussian analogs. Assuming that the distance distribution between any two segments remains Gaussian, the following generalized expressions for an arbitrary Flory-Huggins parameter $\nu$ can be derived for $P(\alpha, n)$ and $A(\alpha, n)$ :

$$
\begin{aligned}
& P(\alpha, n)=2 \int_{0}^{1}(1-u) \exp \left[-\alpha_{\nu} n^{2 \nu} u^{2 \nu}\right] d u, \\
& A(\alpha, n)=\int_{0}^{1} \exp \left[-\alpha_{\nu} n^{2 \nu} u^{2 \nu}\right] d u .
\end{aligned}
$$


In practice, the fitting routine involves the products $(n l)$ and $\left(n^{\prime} l^{\prime}\right)$ as the only parameters. Again, the pair distance distribution can be obtained by numerical Fourier transformation of the form factor.

\section{EXPERIMENT SECTION}

\section{A. Samples}

The star-burst PAMAM (polyamidoamine) dendrimers with generations between $g=0$ and $g=8$ were purchased from Dendritech, Inc. in methanol solution. They were synthesized via reiterative reaction sequences from an ethylenediamine core with functionality $f=4$. Using protection/ deprotection strategies, a new sequence (generation) can be added consisting of amine-amide branching units with functionality $m=2$. The final generation terminates with primary amine endgroups. A more detailed description of the synthesis is given in Refs. 1 and 45.

\section{B. Small-angle x-ray scattering (SAXS)}

The experiments were performed in single-bunch mode at the High Brilliance Beamline ID2 at the European Synchrotron Radiation Facility (ESRF), Grenoble, France. We used a CCD detector with a high spatial resolution of 0.17 $\mathrm{mm}$ and an active area of $230 \mathrm{~mm}$. The spot size on the sample was $(0.3 \times 0.3) \mathrm{mm}^{2}$. At a wavelength of $\lambda=0.1 \mathrm{~nm}$ with a bandwidth of $\Delta \lambda_{\mathrm{FWHM}} / \lambda=2 \times 10^{-4}$, the evaluated $q$ range was $0.08 \mathrm{~nm}^{-1} \leqslant q \leqslant 3.5 \mathrm{~nm}^{-1}$ using detector setting at $d=2$ and $10 \mathrm{~m}$. We used quartz capillaries with a diameter of $1.5 \mathrm{~mm}$ as sample containers. The two-dimensional data were converted to absolute scattering cross sections via standard correction procedures for absorption, detector sensitivity, and background stemming from the deuterated solvent (methanol) and empty cell. In a subsequent step the corrected data were radially averaged. The convolution of the measured spectra with the experimental resolution is considered in the fit routines, even though the effect of the finite $q$ resolution is rather small. The angular resolution of ID2 is $\Delta \theta=500$ and $100 \mu \mathrm{rad}$ at $d=2$ and $10 \mathrm{~m}$, respectively.

\section{Neutron spin-echo (NSE) spectroscopy}

The high-resolution inelastic scattering experiments were carried out on the neutron spin-echo (NSE) spectrometer at the DIDO reactor, Jülich, Germany as well as on the NSE spectrometer MESS at the Orphee reactor of the Laboratoire Léon Brillouin, Saclay, France.

At the NSE spectrometer in Jülich the momentum transfer covered was $0.26 \mathrm{~nm}^{-1} \leqslant q \leqslant 1.80 \mathrm{~nm}^{-1}$, using four different detector arm settings $q=(0.5,0.8,1.0,1.4) \mathrm{nm}^{-1}$. The multidetector allowed the extraction of three to four different adjacent $q$ values within an overall detector range of $\pm 0.27 \mathrm{~nm}^{-1}$ per setting. Using a neutron wavelength of $\lambda$ $=0.8 \mathrm{~nm}$ with a bandwidth of $\Delta \lambda_{\mathrm{FWHM}} / \lambda=0.10$, the $q$ resolution

$$
\Delta q_{\mathrm{FWHM}}=\sqrt{\left(0.1 \mathrm{~nm}^{-1}\right)^{2}+\left(q \times \Delta \lambda_{\mathrm{FWHM}} / \lambda\right)^{2}}
$$

varied between 0.1 and $0.2 \mathrm{~nm}^{-1}$ with $q$. With 14 Fourier time settings, a time range between $0.1 \mathrm{~ns} \leqslant t \leqslant 22 \mathrm{~ns}$ was

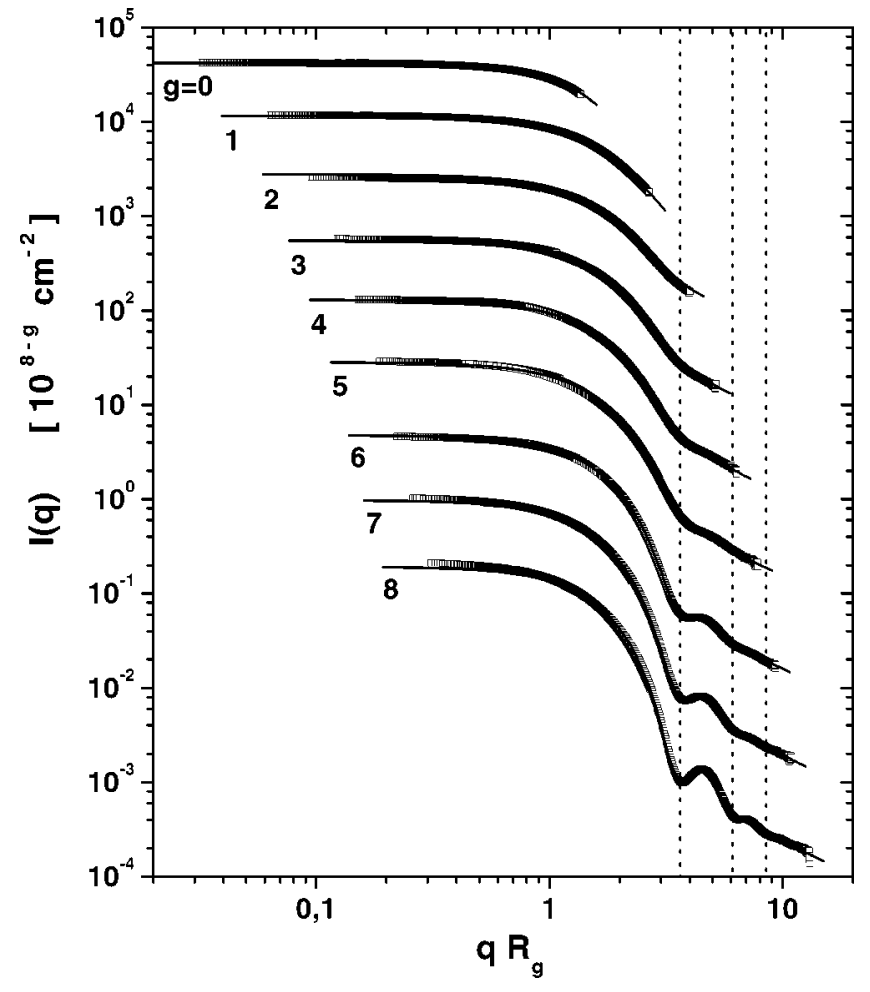

FIG. 2. SAXS spectra for PAMAM dendrimers of generations $g=0$ to $g$ $=8$ in methanol are plotted in a double logarithmic representation. For each generation the $q$ axis is scaled with $R_{g}$ determined by IFT (or Guinier fit) of the SAXS data. Data are separated by a multiplicative constant $10^{8-g}$ for better visibility. The solid lines represent fits of the spectra using the fuzzy sphere model.

covered. The reduction of the multidetector data to $S(q, t) / S(q)$ followed the procedures described in Ref. 46 . As sample containers we used rectangular, sealed niobium cuvettes with a sample thickness of $4 \mathrm{~mm}$ and an illuminated sample area of $(30 \times 30) \mathrm{mm}^{2}$. For background correction, the signal from the deuterated solvent methanol was measured over the full time range and subtracted from the sample signal with the proper correction factors.

The measurements at the Saclay instrument were performed with a $q$ resolution of about $20 \%$ for the fol-

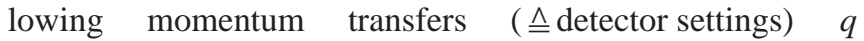
$=(0.366,0.457,0.548,0.640,0.731,0.822,0.914,1.005$, $1.096) \mathrm{nm}^{-1}$. The neutron wavelength was set to $\lambda$ $=0.6 \mathrm{~nm}$ with a wavelength spread of $\Delta \lambda_{\mathrm{FWHM}} / \lambda=0.18$. With 14 Fourier time settings, a time range of $0.25 \mathrm{~ns} \leqslant t$ $\leqslant 17.54$ ns was covered. We used rectangular, sealed quartz cuvettes with a sample thickness of $6 \mathrm{~mm}$ as sample containers. A sample area of $(27 \times 27) \mathrm{mm}^{2}$ was illuminated. The background correction was performed following the procedures described in detail in Ref. 47.

\section{RESULTS AND DISCUSSION}

\section{A. SAXS-Structural properties}

In Fig. 2 the SAXS data obtained for the dendrimers are shown in a double logarithmic representation for all generations $g=0$ to $g=8$. The solid lines give the results obtained from a fit of the data with the fuzzy edge model as discussed 
TABLE I. Summary of the results obtained from the SAXS data by inverse Fourier transformation and from various model fits.

\begin{tabular}{|c|c|c|c|c|c|c|c|}
\hline \multirow[b]{2}{*}{$g$} & \multirow{2}{*}{$\begin{array}{c}\mathrm{IFT} \\
R_{g} \\
{\left[10^{-1} \mathrm{~nm}\right]}\end{array}$} & \multicolumn{2}{|c|}{ Polydisperse sphere } & \multicolumn{2}{|c|}{ Rotational ellipsoid } & \multicolumn{2}{|c|}{ Fuzzy edge } \\
\hline & & $\begin{array}{c}R_{s} \\
{\left[10^{-1} \mathrm{~nm}\right]}\end{array}$ & $\begin{array}{c}\sigma_{s} / R_{s} \\
{[\%]}\end{array}$ & $\begin{array}{c}R_{g} \\
{\left[10^{-1} \mathrm{~nm}\right]}\end{array}$ & $\epsilon$ & $\begin{array}{c}R_{f} \\
{\left[10^{-1} \mathrm{~nm}\right]}\end{array}$ & $\begin{array}{c}\sigma_{f} / R_{f} \\
{[\%]}\end{array}$ \\
\hline 0 & $4.0 \pm 0.3$ & $5.16 \pm 0.010$ & (22) & $4.369 \pm 0.006$ & $(0.516)$ & $4.621 \pm 0.009$ & $(55)$ \\
\hline 1 & $7.9 \pm 0.1$ & $10.24 \pm 0.01$ & (21) & $8.03 \pm 0.01$ & $(0.526)$ & $8.170 \pm 0.006$ & $(54)$ \\
\hline 2 & $11.8 \pm 0.2$ & $15.22 \pm 0.01$ & $20.0 \pm 0.2$ & $11.80 \pm 0.04$ & $0.536 \pm 0.004$ & $11.68 \pm 0.03$ & $53 \pm 3$ \\
\hline 3 & $15.09 \pm 0.05$ & $19.47 \pm 0.02$ & $19.0 \pm 0.2$ & $15.05 \pm 0.04$ & $0.547 \pm 0.004$ & $15.53 \pm 0.02$ & $52 \pm 2$ \\
\hline 4 & $18.60 \pm 0.07$ & $24.35 \pm 0.04$ & $18.2 \pm 0.1$ & $18.62 \pm 0.04$ & $0.554 \pm 0.003$ & $19.64 \pm 0.04$ & $50 \pm 2$ \\
\hline 5 & $23.07 \pm 0.03$ & $29.95 \pm 0.08$ & $17.3 \pm 0.2$ & $22.76 \pm 0.09$ & $0.580 \pm 0.005$ & $24.37 \pm 0.04$ & $48 \pm 1$ \\
\hline 6 & $27.50 \pm 0.03$ & $35.54 \pm 0.05$ & $12.71 \pm 0.09$ & $27.22 \pm 0.05$ & $0.668 \pm 0.003$ & $32.04 \pm 0.04$ & $28.4 \pm 0.4$ \\
\hline 7 & $32.11 \pm 0.05$ & $41.67 \pm 0.05$ & $11.50 \pm 0.08$ & $31.92 \pm 0.06$ & $0.698 \pm 0.003$ & $38.89 \pm 0.09$ & $25.9 \pm 0.6$ \\
\hline 8 & $38.58 \pm 0.10$ & $49.79 \pm 0.07$ & $9.80 \pm 0.09$ & $38.20 \pm 0.05$ & $0.739 \pm 0.002$ & $47.50 \pm 0.08$ & $21.9 \pm 0.6$ \\
\hline
\end{tabular}

below. For better visibility the data are separated by a factor $10^{(8-g)}$. The absolute SAXS intensities are plotted versus the generalized variable $q R_{g}$ to allow a direct visualization of changes in the general shape between particles independent of their dimensions. For $g \geqslant 2$ the radii of gyration $R_{g}$ are obtained from inverse Fourier transformation of the SAXS data following the procedures described below and in Sec. II B 1 and for $g \leqslant 2$ by a Guinier fit of the low- $q$ region. The results for $R_{g}$ obtained from these procedures are listed in Table I. The intensities measured at low polymer concentrations $(\Phi=1 \mathrm{wt} \%)$ represent the single-particle form factor, since further dilution did not result in a change of the spectra, indicating that interparticle interaction can be neglected. To ensure the best possible background correction, the data in the high- $q$ range were obtained from samples with higher polymer concentrations so that the signal was always 4 times larger than the background.

For dendrimers of generation $g=6$ to $g=8$, secondary and ternary $(g=8)$ maxima are clearly visible, supporting the picture of a spherical shape for high-generation dendrimers. The minima at $q_{\min } R_{g}=3.70,6.12,8.55$ which are most sensitive to measure the size of a spherical particle are again in very good agreement with the values expected for homogeneous spheres: $q_{\min } R_{g}=\sqrt{3 / 5}(2 n+1) \pi / 2=3.65$, $6.08,8.52$, with $n$ being a positive integer. In the high- $q$ range $\left(q R_{g} \geqslant 3.5\right)$, the scattering from the (loose) internal polymeric structure called "blob scattering" becomes visible, and is especially evident for the low dendrimer generations $g \leqslant 5$. The scattering intensity follows a $q^{-5 / 3}$ powerlaw dependence as would have been expected for linear polymers under good solvent conditions. For higher generations, the blob scattering leads to a smearing out of the minima of the sphere form factor. These observations become even more pronounced when the absolute intensities versus $q R_{g}$ are plotted in a generalized Kratky representation, as shown in Fig. 3. For the high-generation dendrimers $(g \geqslant 6)$, the first maximum in the Kratky plot appears at $1.48 \geqslant q R_{g} \leqslant 1.51$, which compares very well with the value of $q R_{g}=1.49$ expected for homogeneous spheres. With decreasing generation the peak shifts towards somewhat higher values up to about $q R_{g} \approx 1.66$ for $g=3$.

The lowest generation for which we are able to resolve not only the overall shape of the dendrimer but also a sig- nificant part of the blob contribution in the $q$ range of our experiment is the $g=3$ dendrimer. In Fig. 4 a comparison of the experimental SAXS spectrum obtained for $g=3$ with the theoretical prediction for the form factor of a hard solid sphere [Eq. (29) with $r^{\prime}=R_{s}$ ] and the form factor of an analogous dendrimer under good solvent conditions [Eqs. (32)-(35)] are shown. In addition, we calculated the form factor of a star with 4 and 32 branches corresponding to the number of dendrons and terminal units of the $g=3$ dendrimer, respectively. The star form factor is a special case $(g=1)$ of the dendrimer form factor. The sphere radius and segment length are chosen in such a way that the radius of

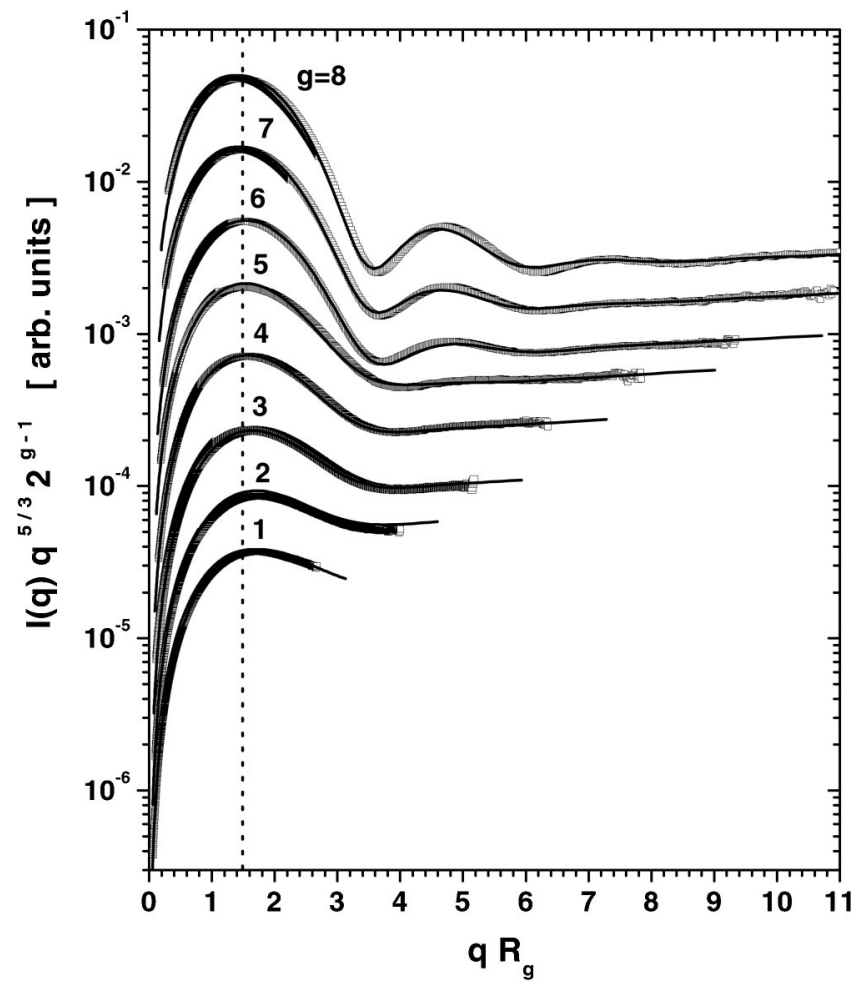

FIG. 3. SAXS spectra for PAMAM dendrimers of generation $g=0$ to $g$ $=8$ in methanol in semilogarithmic Kratky representation generalized to good solvent conditions. For each generation the $q$ axis is scaled by $R_{g}$ determined by IFT (or Guinier fit) of the SAXS data. Data are separated by a multiplicative constant $2^{g-1}$ for visibility. The solid lines represent fits of the spectra using the fuzzy sphere model. 


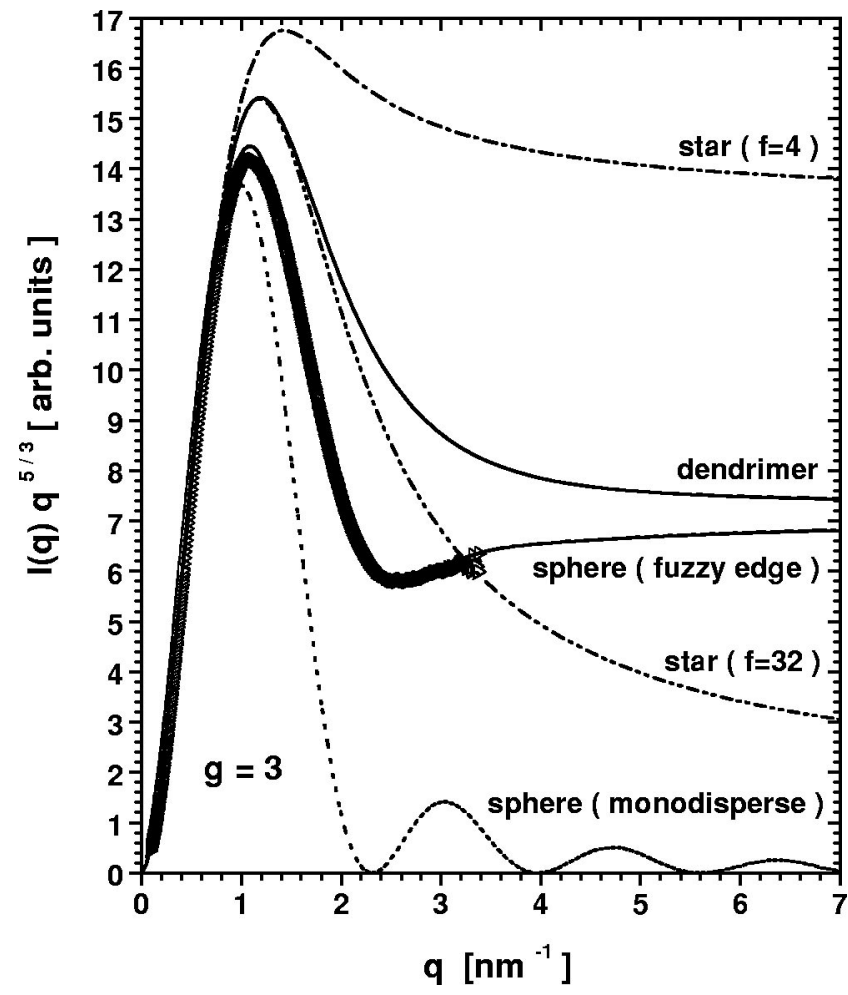

FIG. 4. Generalized Kratky representation of the SAXS spectrum for $g$ $=3$ (markers) compared with the theoretical predictions for star polymers $(f=4$ and 32) and a dendrimer $(g=3, f=4, m=2)$ under good solvent conditions. In addition, the theoretical results for a homogeneous sphere and for a fuzzy sphere with internal density fluctuations are shown.

gyration is equal to the value obtained from the IFT of the experimental data. The number of segments was fixed to be equal to that of the dendrimer. It is obvious that in the intermediate $q$ region the decay of the measured dendrimer form factor with $q$ is much steeper than predicted from the theories for the different polymer architectures (star and dendrimer), but weaker than predicted for a hard solid sphere. Even though the evaluable $q$ region (before the blob scattering comes into play) is too small for a correct determination of the fractal dimension $P$ of the $g=3$ dendrimer, we determined $P$ from the decay of the form factor $\left(\propto q^{-P}\right)$ in the intermediate $q$ region to be close to $P=4$, and definitely much larger than $P=3$ predicted for high-functionality stars. Therefore, we conclude that even the low-generation dendrimers are surface fractals rather than mass fractals.

Note that for an ideal homogeneous sphere, $P(q)$ would decay faster than $q^{-4}$ in this intermediate $q$ range, as can be seen in Fig. 4. In principle, we can imagine three different scenarios which would lead to a reduced slope in the intermediate $q$ region and to a smearing of the minima of the sphere form factor in addition to the smearing caused by the blob scattering contribution: (1) the dendrimers are polydisperse, homogeneous spheres; (2) their shape is ellipsoidal rather than spherical or, last but not least (3) their density profile is not perfectly sharp but shows a fuzzy, outer surface region. For more details on the different models see Sec. II C 1.

In principle, all three models lead to a description of the data of similar quality with consistent results summarized in
Table I. The only difference is that the model of the rotational ellipsoid as well as the model of the spheres with Gaussian form polydispersity lead to a rather small effective blob radius of $\bar{\xi}=(0.6 \pm 0.1) \mathrm{nm}$, which would contain only about four segments, whereas the fuzzy edge model gives a value of $\bar{\xi}=(1.5 \pm 0.1) \mathrm{nm}$ with about 22 segments [determined from Eq. (27); see the discussion below]. From previous publications on high-functionality stars, we consider the latter result to be more reasonable. ${ }^{40}$ In addition, with only four segments contributing to the blob scattering the normal picture of chain statistics, based on a random coiled structure, might not be applicable. Therefore, we would like to focus the following discussion on the fuzzy edge model.

For the $g=8$ dendrimer in Fig. 1 the different contributions to the overall scattering stemming from the blob scattering $P(q)_{\text {blob }}$ and from the globular shape $P(q)_{\text {shape }}^{f_{s}}$ (with fuzzy edge of relative width $\sigma_{f} / R_{f}=21.7 \%$ ) are plotted separately. All curves incorporate instrumental resolution effects, which even though very small are considered in the fit routines. For the highest generations we are most sensitive to distinguish between effects rising from the blob scattering and the detailed character of the surface, respectively. In a separate fit for each generation $(g=6,7,8)$, leaving all parameters including the Flory-Huggins parameter $\nu$ adjustable, it turned out that neither $\nu$ nor $\bar{\xi}$ changes significantly and/or systematically around the average values $\nu_{\mathrm{av}}=1.6$ \pm 0.1 and $\bar{\xi}_{\mathrm{av}}=(1.5 \pm 0.1) \mathrm{nm}$. Therefore, we kept $\bar{\xi}$ fixed to its average value and the Flory-Huggins parameter was set to its good solvent value of $\nu=3 / 5$. Hence, the only fit parameters left are the radius $R_{f}$, the width of the density decay in the surface region $2 \sigma_{f}$, and the relative amplitude of the blob scattering $a_{b}$. In Fig. 2 and Fig. 3 the curves fitted to the SAXS spectra are represented by the solid lines. Very good agreement between experimental data and fit is obtained for all dendrimer generations.

In the top part of Fig. $5 a_{b}$ is shown as a function of the dendrimer generation. The solid line represents a fit with Eq. (27) with the number of segments $N_{b}$ in one blob as the only parameter. Since the blob size is not a function of $g$, we assume also $N_{b}$ to be independent of the generation. With $N_{b}=21.7 \pm 0.1$ segments per blob, a very good description of the data is achieved. For dendrimers with generations $g \leqslant 2$ we either barely reach or do not access the high- $q$ region where we would be sensitive to the density fluctuations. This can be easily seen from the Kratky representation of the measured SAXS spectra in Fig. 3. For $g=2$ the fit still gives a reasonable result, but for $g \leqslant 1$ we kept $a_{b}$ fixed to the theoretical value which is given in Fig. 5 in brackets.

In the bottom part of Fig. 5 the ratio $\sigma_{f} / R_{f}$ of the (half) width of the surface region relative to the radius $R_{f}$ of the corresponding solid sphere is plotted versus the generation. The exact values are summarized in Table I. Decreasing the generation from $g=8$ to $g=6$ or $g=5$ to $g=3$ results in only a slight extension of the surface region. The relative width $\sigma_{f} / R_{f}$ increases linearly from about $21.9 \%$ to $28.4 \%$ and $48 \%$ to $51 \%$, respectively. Between the sixth and the fifth generation we observe a rather drastic stepwise increase of about $20 \%$. For $g \leqslant 1$ the fit is no longer sensitive to the 


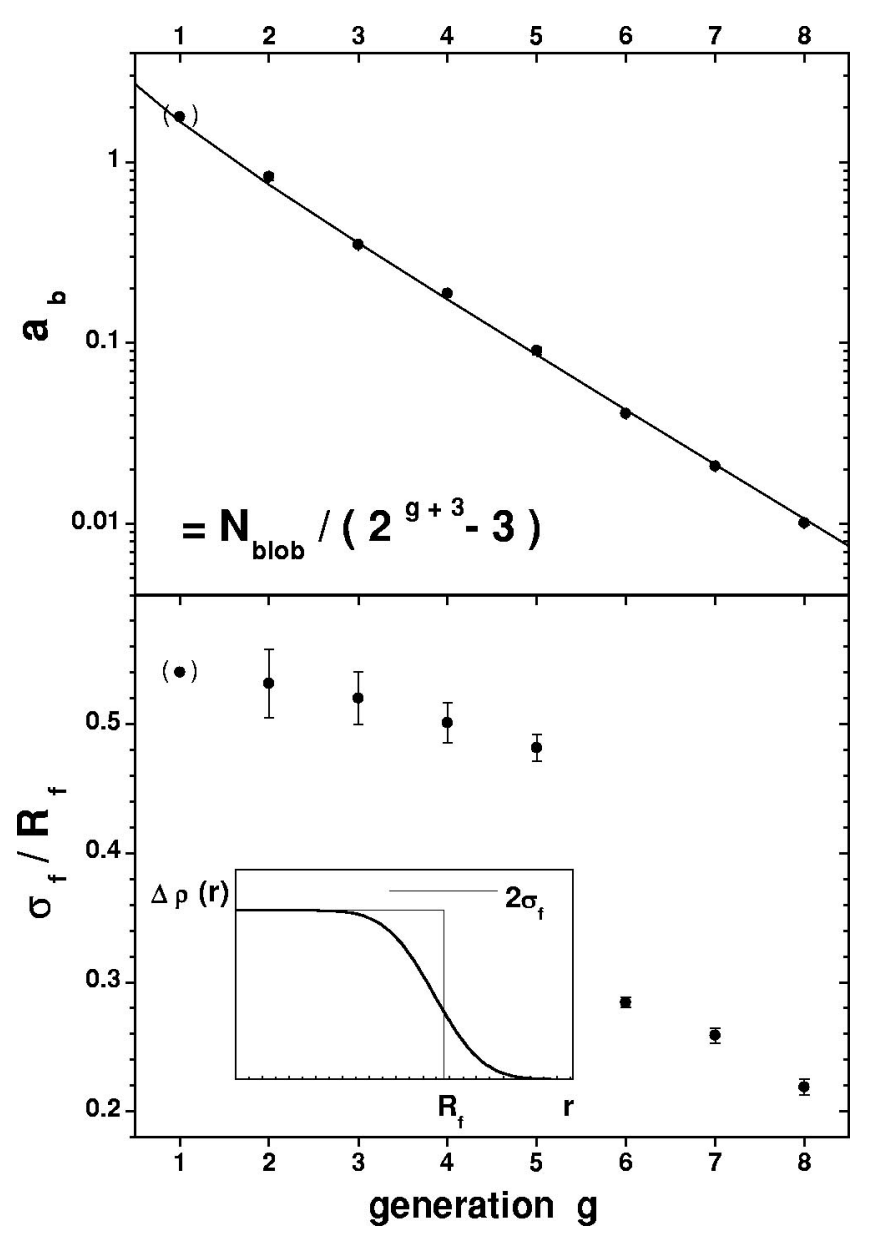

FIG. 5. Results obtained from a fit of the SAXS spectra using the fuzzy sphere model plotted as a function of the generation $g$. Top: Amplitude of the blob scattering relative to the shape contribution. The parenthetical value for $g=1$ was kept fixed in the fit. The solid line shows the dependency expected for a constant number of $N_{\text {blob }}=21.7$ segments per blob. Bottom: Half-width $\sigma_{f}$ of the fuzzy shell region relative to the radius $R_{f}$ of the analogous homogeneous sphere. The inset shows the model parameters and the excess electron density profile obtained for the $g=7$ dendrimer.

width of the surface region. Therefore, we kept $\sigma_{f} / R_{f}$ fixed to the extrapolated values given in Fig. 5 and Table $I$ in brackets.

In Fig. $6, R_{f}$ is plotted versus the dendrimer generation. From the basic relation that the volume $\left(\propto R^{3}\right)$ is proportional to the molecular weight $\propto\left(2^{g+3}-3\right)$ for dendrimers with constant density in the core region, we can derive that $R$ has to follow the curve given by the solid line. The experimental values for $R_{f}$ follow the calculated dependence very well down to the second generation. Only the experimental values obtained for $g=1$ and $g=0$ are smaller than predicted by this simple relation, which would correspond to a higher density if the same overall shape is maintained. In the same figure the radii of gyration obtained by IFT and Guinier fit are also included. The observation that for high generations $R_{g}$ is smaller than predicted by $R \propto\left(2^{g+3}-3\right)^{1 / 3}$ but $R_{f}$ still satisfies the condition can be explained from the definition of the radius of gyration: $R_{g}=\sum_{i=1}^{N}\left\langle r_{i}^{2}\right\rangle / N$, where $N$ is the total number of segments in the molecule and $r_{i}$ are their position vectors measured from the center of gravity. For the dendrimers with generation $g \geqslant 6$, the extension of the sur-

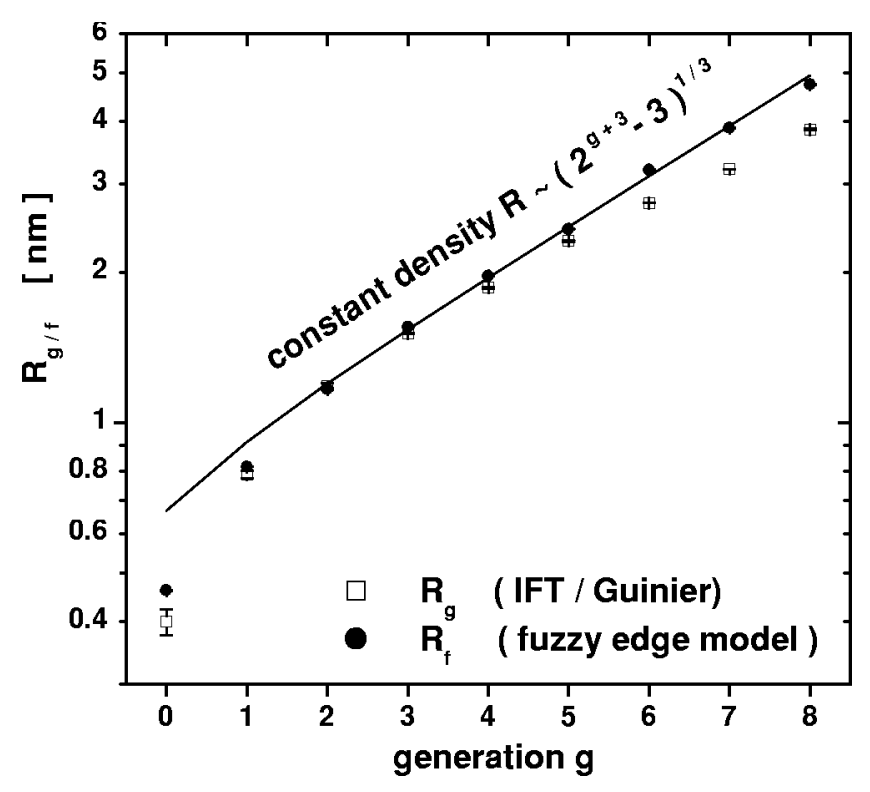

FIG. 6. Radius of gyration $R_{g}$ determined by IFT and Guinier fit and the radius $R_{f}$ obtained by fitting the fuzzy sphere model to the SAXS data as a function of the generation. The solid line shows the result expected for homogeneous spheres with generation-independent density.

face region becomes abruptly smaller and is constantly decreasing up to the eighth generation. A larger number of segments are closer to the center and as a consequence the increase in $R_{g}$ is smaller than for the lower generations. The results for the fit parameters of the sphere with Gaussian form polydispersity and for the rotational ellipsoid are consistent with the findings obtained for the fuzzy edge model (see Table I). The relative form polydispersity $\sigma_{s} / R_{s}$ increases significantly and the aspect ratio (that plays the same role as the polydispersity of the spheres or the fuzziness of the shell region) also shows a stepwise increase to a more asymmetric form between the sixth and fifth generation. The radii of gyration of the ellipsoids are in good agreement with the IFT results. It is interesting that the ratio $R_{g} / R_{s}$, where $R_{s}$ are the sphere radii obtained for the polydisperse spheres, does not change significantly with generation around the average value of $R_{g} / R_{s}=0.772 \pm 0.004$. The latter is close to the theoretical value $\sqrt{5 / 3} \approx 0.775$ for an ideal, homogeneous sphere.

In the following, we would like to discuss the results obtained from the SAXS spectra by inverse Fourier transformation (IFT) and by the square-root deconvolution method (SQDEC) described in more detail in Sec. II B. The main goal of applying the IFT and SQDEC method is to compare the results from this model-independent approach to our results obtained from the model fits described above. Again, we first consider the $g=3$ dendrimer. In Fig. 7 the pair distance distribution function $p(r)$ which gives the probability to find two segments in a distance $r$ from each other in the interior of a single dendrimer is plotted as a function of the intersegmental distance normalized to the radius of gyration $R_{g}$. The data are compared to the theoretical curves for a homogeneous sphere, for a sphere with a fuzzy edge, and for a dendrimer under good solvent conditions. The predictions for a star with functionality $f=4$ and $f=32$ in good solvent 


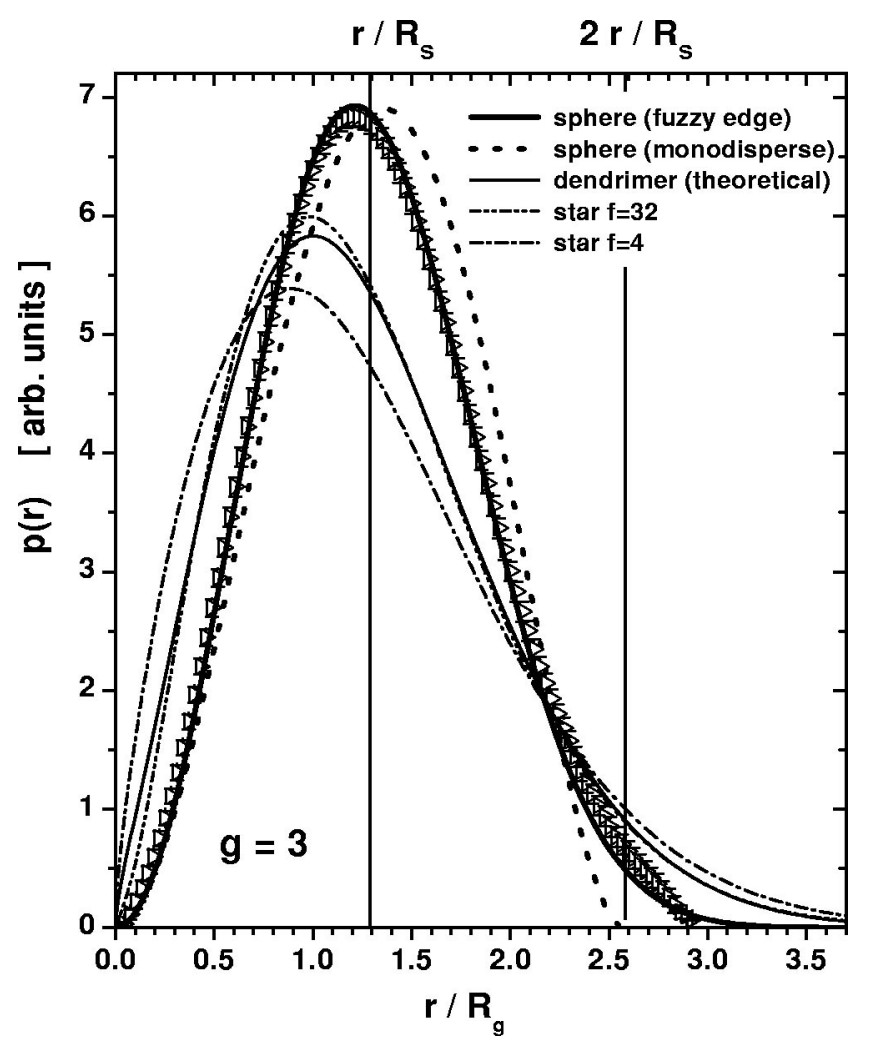

FIG. 7. Comparison of the pair distance distribution function $p(r)$ of the $g=3$ dendrimer determined by IFT of the SAXS data to various theoretical predictions: (a) stars $(f=4$ and 32$)$; (b) dendrimer $(g=3, f=4, m=2)$ in good solvent; (c) homogeneous sphere with sharp edge; and (d) sphere with fuzzy shell region. All curves are normalized to the same radius of gyration $R_{g}$ and molecular weight.

are also included. All curves are normalized to the same $R_{g}$ and the same molecular weight. It is obvious that $p(r)$ for the $g=3$ dendrimer with the exception of a small tail to longer distances is rather symmetric like $p(r)$ of the homogeneous sphere. The small tail and the shift of the peak position to smaller distances are due to the fuzziness of the surface region. The shape of $p(r)$ theoretically calculated for the dendrimer under good solvent conditions as well as of those calculated for the star polymers are much more asymmetric with a much more extended tail to longer distances. In addition, the peak position is shifted to smaller distances of about $r \leqslant R_{g}$ instead of $r \approx 1.18 \times R_{g}$ as found for the $g=3$ dendrimer.

In Fig. $8 p(r)$ obtained by IFT is plotted for dendrimers with generations between $g=2$ and $g=8$. The condition where $r$ is equal to the fitted sphere radius $R_{s}$ and the sphere diameter $\left(2 \times R_{s}\right)$ are marked by solid lines. Note that for an ideal homogeneous sphere we would expect $p(r)$ to have its maximum at $r \approx 1.05 \times R_{s} \approx 1.36 \times R_{g}$. Due to the surface fuzziness, the peak positions (marked by the crosses) are shifted to smaller distances for all dendrimer generations. The shift becomes increasingly larger for $g \leqslant 6$ and varies from $1.30 \times R_{g}$ for the $g=8$ dendrimer to $1.13 \times R_{g}$ for the $g=2$ dendrimer. The solid lines represent the theoretical curves calculated from the form factor of the fuzzy sphere by inverse Fourier transformation [see Eq. (13)]. The parameters $\sigma_{f} / R_{f}, R_{f}, \bar{\xi}$, and $a_{b}$ are taken as previously deter-

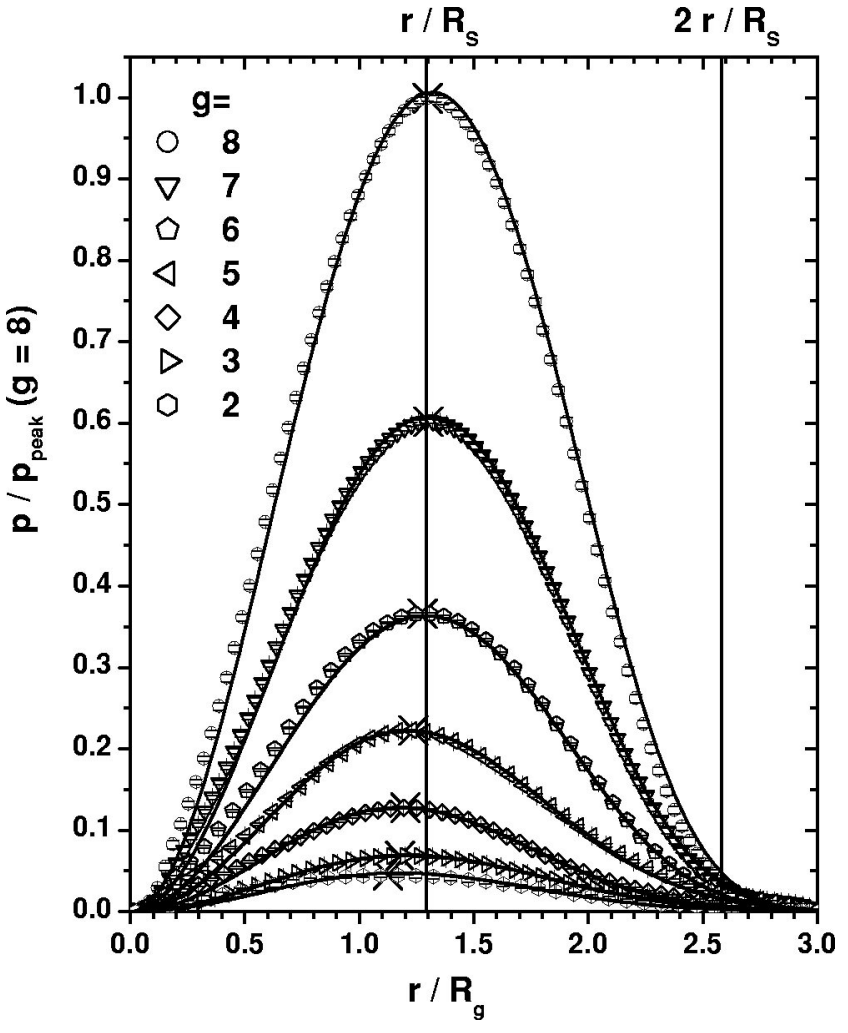

FIG. 8. Pair distance distribution function $p(r)$ determined by IFT from the SAXS data for dendrimer generations $g=2$ to 8 as a function of the normalized intersegmental distance $r / R_{g}$. The $y$ axis is normalized to the peak value of the $g=8$ dendrimer. The peak positions are marked by the crosses. The solid lines represent the predictions of the fuzzy sphere model calculated with the parameters previously determined by the fit of the SAXS spectra.

mined so that there are no free parameters left. Very good agreement is achieved for all dendrimer generations.

The excellent agreement between our fit results and the results obtained by the model-independent IFT approach is further outlined in Fig. 9, where the density profiles are plotted as a function of the radial distance $r$ from the center normalized to the radii of gyration $R_{g}$ for each generation. We normalized the experimentally determined absolute excess electron density $\Delta \rho_{\text {exp }}$ to its theoretical value $\Delta \rho_{\text {theo }}$ $=3.63 \cdot 10^{10} \mathrm{~cm}^{-2}$, which has been calculated from the electron densities and the bulk densities ${ }^{48}$ of the pure components. $\Delta \rho_{\exp } / \Delta \rho_{\text {theo }}$ gives the polymer volume fraction $\Phi_{P}$ in each subshell. The solid lines show the curves determined by inverse Fourier transformation of the amplitude [see Eq. (31)] of the theoretical scattering intensity calculated in the framework of the fuzzy edge model. Again, we kept all parameters fixed to the previously determined fit results. The agreement between both approaches is excellent within the error bars of the SQDEC method.

The most significant conclusions we would like to draw from this section which are of vital importance for the interpretation of the NSE data are the following: Down to the third generation the dendrimers have a rather globular, compact shape. Although the shell region with a smoothly decaying density profile becomes increasingly larger for the lowergeneration dendrimers, the steep decay of $P(q)$ in the 


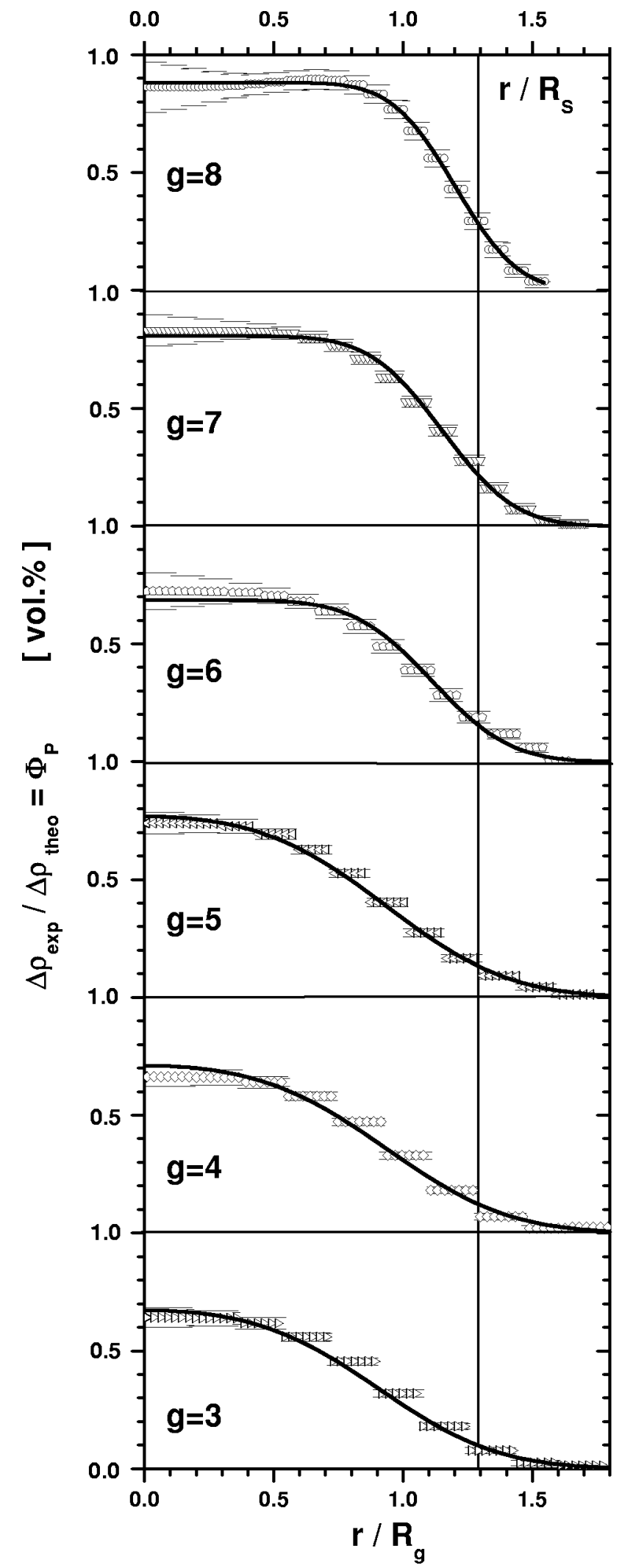

FIG. 9. Segment density profiles determined by SQDEC and IFT from the SAXS data for dendrimer generations $g=3$ to $g=8$ as a function of the normalized radial distance to the center $r / R_{g} . \Phi_{P}(r)$ as a measure for the polymer volume fraction was calculated from the ratio of the experimentally determined excess electron density to its theoretical value. The solid lines represent the predictions of the fuzzy sphere model calculated with the parameters previously determined by a fit of the SAXS spectra.

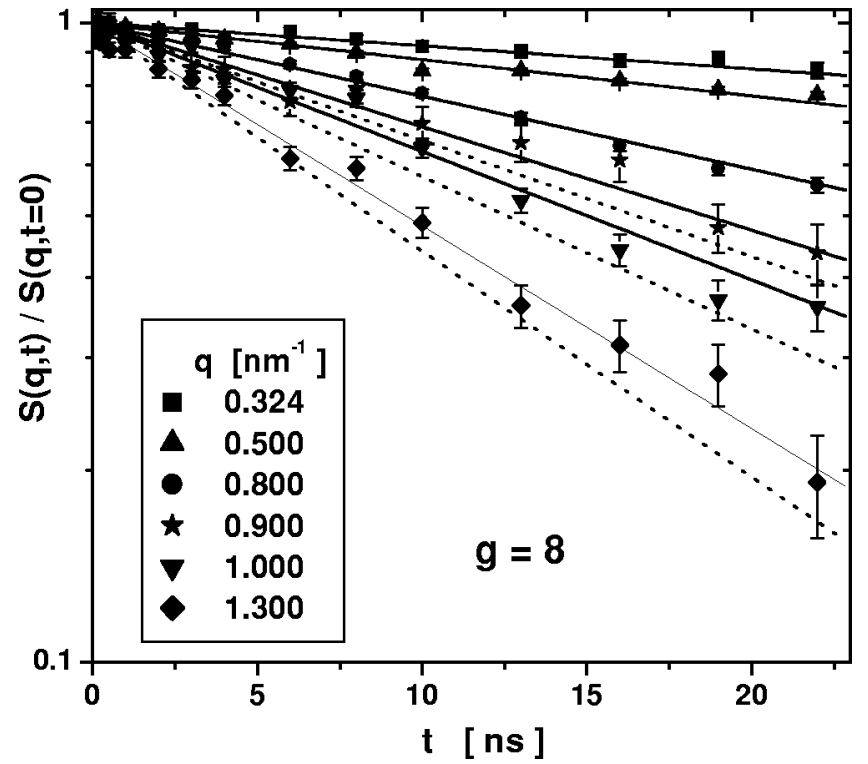

FIG. 10. NSE spectra measured for the $g=8$ PAMAM dendrimer in deuterated methanol solution at a concentration of $5 \mathrm{wt} \%$. The solid lines correspond to a fit with a single exponential decay for each $q$ value separately. To answer the question how sensitive are the NSE measurements to internal relaxation, the dashed lines represent the results using Eq. (39).

intermediate- $q$ region indicates that down to $g=3$ the dendrimers are surface fractal rather than mass fractals. Between the sixth and fifth generation, we observe a major change in the characteristics of the density profile- the extension of the shell region compared to the overall dimension of the dendrimer increases abruptly.

\section{B. NSE-Dynamic properties}

Depending on the chosen scattering vector range, neutron spin-echo (NSE) spectroscopy can resolve the polymer dynamics on different length scales. The $q$ range investigated in our experiment covers length scales much smaller than the radius of gyration, as well as length scales larger than the overall dimensions of the dendrimers. Measurements were performed on dendrimers with generations between $g=5$ to $g=8$ in deuterated methanol at a polymer concentration of $\Phi=5 \mathrm{wt} \%$ and a temperature of $T=293 \mathrm{~K}$. In Fig. 10 the spectrum obtained for the $g=8$ dendrimer is shown in a semilogarithmic plot showing five representative $q$ values. It is obvious that the intermediate structure factor exhibits a single exponential decay in the whole $q$ - and time range. The solid lines correspond to fits to single exponential functions for each $q$ value separately from which the first cumulant $\Omega(q)$ or the relaxation time $\tau(q)=\Omega^{-1}(q)$ can be determined. Limiting the fit to shorter time ranges does not significantly change the results for the relaxation times. For intensity reasons the NSE measurements were performed at a polymer concentration where for the smallest $q$ values $q$ $\leqslant 0.4 \mathrm{~nm}^{-1}$ the structure factor influence due to interparticle interaction cannot be neglected. In the following, the intrinsic relaxation rates are corrected for this effect via the relation: 


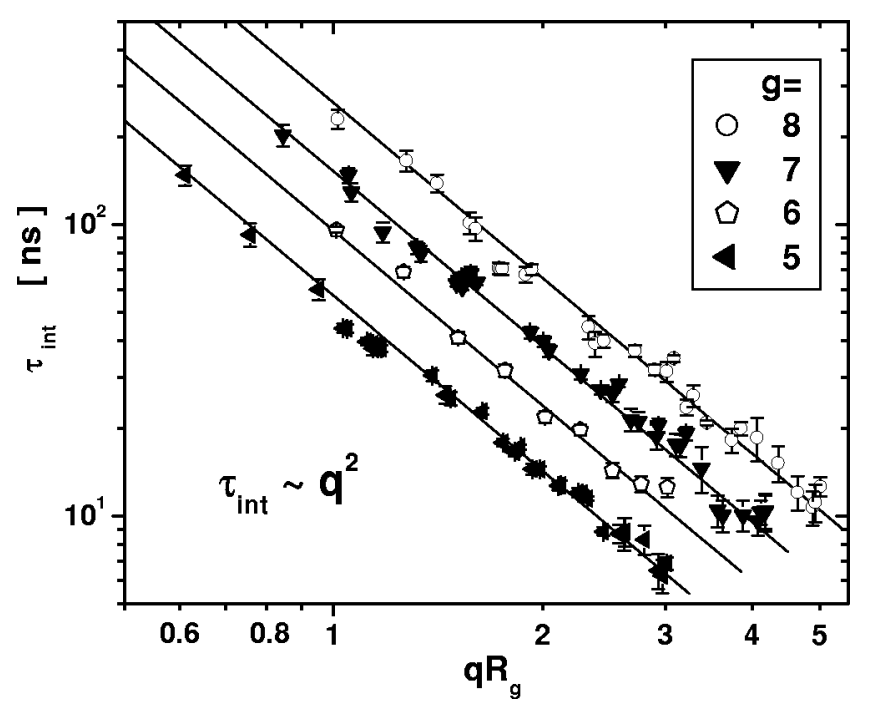

FIG. 11. Relaxation rates obtained from a single exponential fit of the NSE signal for PAMAM dendrimers with generation $g=5$ to $g=8$ as a function of the generalized variable $q R_{g}$ in a double-logarithmic representation. The solid lines show the $q^{-2}$ dependence predicted for simple Stokes-Einstein diffusion of hard spheres at finite concentration.

$$
\tau_{\text {int }}(q)=\tau_{\text {obs }}(q) \frac{P(q)}{S_{\text {obs }}(q)},
$$

where $P(q)$ is the dendrimer form factor obtained from the low-concentration (1 wt \%) SAXS data. $S_{\text {obs }}(q)$ denotes the observed static spectra measured for samples with a dendrimer concentration of $5 \mathrm{wt} \%$ for which the observed relaxation rates $\tau_{\text {obs }}(q)$ have been determined in the NSE experiment. The ratio $S_{\text {obs }}(q) / P(q)$ gives the structure factor which describes the interdendrimer interaction. Figure 11 shows a double-logarithmic representation of the fit results for the (intrinsic) relaxation rates versus the generalized variable $q R_{g}$ for all dendrimer generations. The radii of gyration are obtained from IFT of the corresponding SAXS spectra. For all dendrimer generations the relaxation rates follow a $q^{-2}$ power-law decay. The solid lines correspond to a fit to such a power-law decay. As outlined in Sec. II A a $q^{-2}$ dependence of the relaxation rates indicates a simple diffusional process. For center-of-mass diffusion of ideal, homogeneous spheres, the Stokes-Einstein relation with correction for finite concentration can be derived, leading to the following equation for the diffusion coefficient $D$ :

$$
D=\left(q^{2} \tau\right)^{-1}=\frac{k_{B} T}{6 \pi \eta_{s} R_{s}}(1-k \Phi),
$$

where $\eta_{s}$ is the viscosity of the solvent, $\Phi$ is the volume fraction of the dendrimer, and $R_{s}$ is the sphere radius which might be replaced for an arbitrary particle by the hydrodynamic radius $R_{h}$. For ideal hard-sphere interaction $k$ can be calculated to be equal to $2.1 .^{49}$ In Fig. 12 the inverse of $q^{2} \tau_{\text {int }}$ normalized to the sphere radii $R_{s}$ is plotted as a function of $q$. The sphere radii $R_{s}$ are taken from the model fit of the SAXS data with the form factor of polydisperse spheres (see above). As expected for simple center-of-mass diffusion, in this representation the data for all dendrimer generations superimpose to a horizontal line within the error bars of the

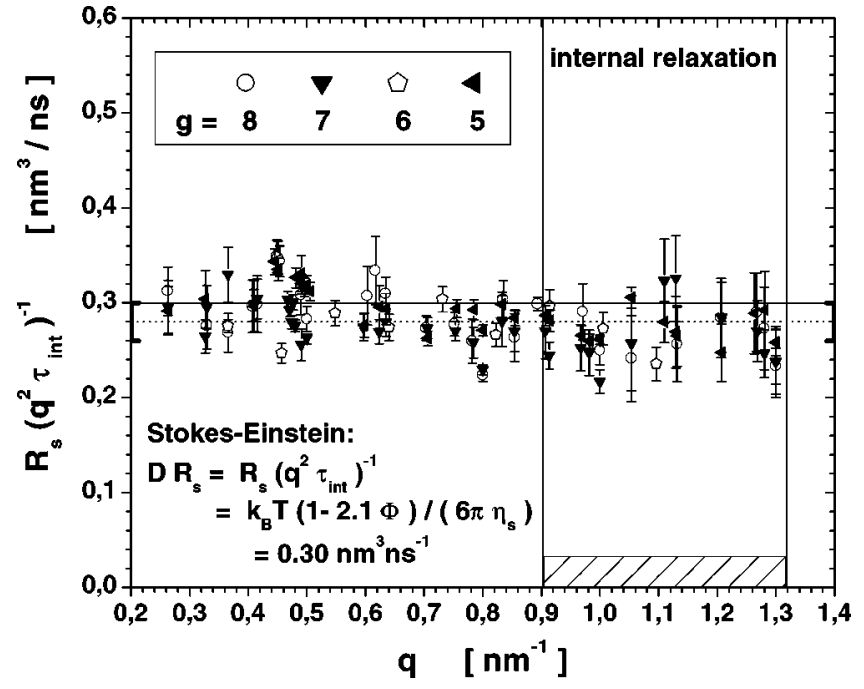

FIG. 12. Diffusion coefficients scaled with the sphere radii $\left(D \times R_{s}\right)$ as a function of the scattering vector $q$ for dendrimers with generation $g=5$ to $g=8$. The $R_{s}$ were obtained from a fit of the SAXS spectra using the polydisperse sphere model. The solid line represents the condition for simple Stokes-Einstein diffusion of hard spheres at finite concentration. The dashed line marks the constant obtained by fitting all experimental relaxation rates simultaneously.

method. Insertion of $\eta_{s}=0.65 \mathrm{mPas}$ for deuterated methanol, $T=293 \mathrm{~K}$, and $\Phi=3.69 \mathrm{vol} \%$ into Eq. (38) leads to $R_{s} /\left(q^{2} \tau_{\text {int }}\right)=0.30 \mathrm{~nm}^{3} / \mathrm{ns}$, which compares very well to the value of $(0.28 \pm 0.02) \mathrm{nm}^{3} / \mathrm{ns}$ obtained by fitting all experimental relaxation rates simultaneously. Since NSE rather measures the "true" hydrodynamic radii, but the theoretical value calculated from the sphere radii $R_{s}$ gives the diffusion coefficients of the corresponding hard solid spheres, we obtain: $R_{h}=(0.94 \pm 0.07) \times R_{s}$. The values of the measured diffusion coefficients are summarized in Table II.

Therefore, we would like to conclude at this point: Even though we are also covering length scales smaller than the dendrimer dimension, we do not see any contribution of internal relaxation processes to the decay of the dynamic structure factor. Within the error bars of the method the hydrodynamic radii $R_{h}$ obtained from the short-time center-of-mass diffusion coefficient equal the hard-sphere radii $R_{s}$ obtained from the static SAXS investigations. Dendrimers with generations from $g=5$ to $g=8$ show simple Stokes-Einstein diffusion of hard spheres.

We now consider which $q$ range would yield sufficient signal from the internal structure to be sensitive to internal relaxation processes. In Fig. 13, the relative contribution of the blob scattering $P(q)_{\text {blob }}$ to the contribution of the overall scattering $P(q)$ is shown as a function of the scattering vector. It is obvious that for scattering vectors up to $q$ $\approx 0.9 \mathrm{~nm}^{-1}$ the major contribution to the scattering stems

TABLE II. Diffusion coefficients obtained from NSE spectroscopy for PAMAM dendrimers in deuterated methanol.

\begin{tabular}{ccccc}
\hline \hline $\mathrm{g}$ & 5 & 6 & 7 & 8 \\
\hline $\mathrm{D}\left[10^{-7} \mathrm{~cm}^{2} / \mathrm{s}\right]$ & $9.4 \pm 0.4$ & $7.9 \pm 0.3$ & $6.8 \pm 0.2$ & $5.7 \pm 0.2$ \\
\hline \hline
\end{tabular}




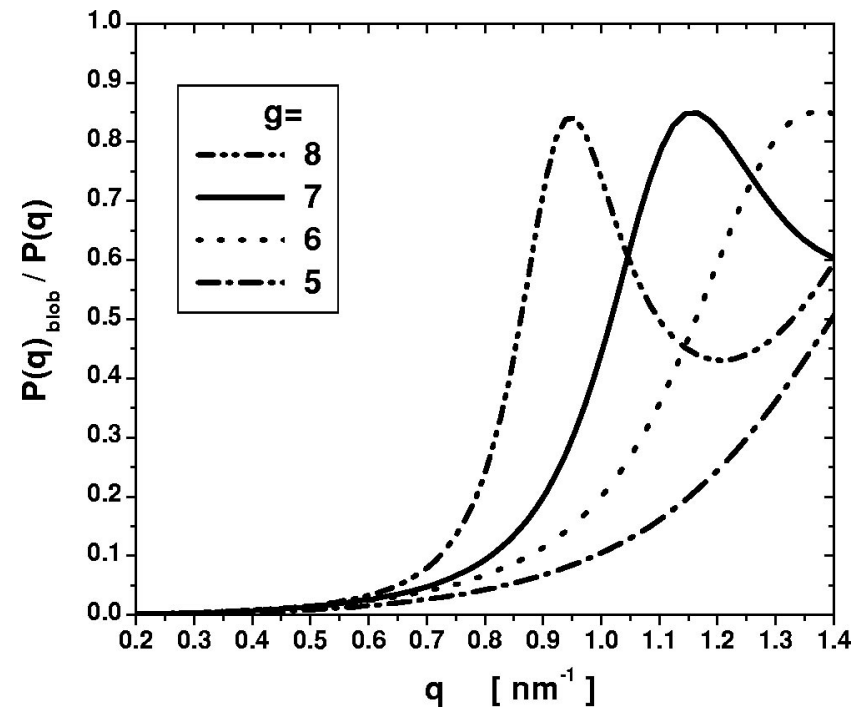

FIG. 13. Relative contribution of the blob scattering $P_{\text {blob }}(q)$ to the overall scattering as a function of the scattering vector $q$ for dendrimers with generation $g=5$ to $g=8 . P(q)_{\text {blob }}$ and $P(q)$ were calculated using the fit results of the fuzzy sphere model.

from the overall dendrimer shape, and therefore only centerof-mass diffusion, can be observed. For scattering vectors larger then $q \approx 0.9 \mathrm{~nm}^{-1}$ the internal relaxation should give a significant contribution to the relaxation of the dynamic structure factor at least for the $g=7$ and $g=8$ dendrimers. To test how sensitive the NSE measurements are to internal relaxation, we made the following ansatz:

$$
\begin{aligned}
\frac{S(q, t)}{S(q, 0)}= & {\left[1-\frac{P(q)_{\text {blob }}}{P(q)-P(q)_{\text {blob }}}\right] \exp \left(-t / \tau_{\mathrm{cm}}\right) } \\
& +\frac{P(q)_{\text {blob }}}{P(q)-P(q)_{\text {blob }}} \exp \left(-t / \tau_{\mathrm{cm}}\right) \exp \left(-t / \tau_{\text {int }}\right)
\end{aligned}
$$

where $\tau_{\mathrm{cm}}$ and $\tau_{\text {int }}$ are the relaxation times of the center-ofmass diffusion and the internal relaxation dynamics, respectively. In Fig. 10 the dotted lines mark the results for $\tau_{\text {int }}$ $=4 \times \tau_{\mathrm{cm}}$ under the assumption that the contribution of the blob scattering to the overall scattering is only $50 \%$. It is obvious that internal relaxations which are 4 times slower than the center-of-mass diffusion still give a significant contribution to the decay of the dynamic structure factor. We can therefore conclude that the internal relaxation must be at least 4 times slower than the diffusion of the overall dendrimer.

If we compare these results to those obtained on highfunctionality star polymers by Richter et al., ${ }^{18-20}$ our observations suggest that the internal dendrimer dynamics is much slower (at least one order of magnitude) than the internal relaxation of the star polymers. The star polymers under investigation had diffusion coefficients of the same order (or even faster) as the dendrimers studied here. As shown in Sec. II A the relaxation rates for the center-of-mass diffusion increase with a $q^{2}$ dependence, but the internal Zimm dynamics becomes faster with $q^{3}$. Therefore, the internal dynamics should dominate the decay of the dynamic structure fac- tor at high scattering vectors and the relaxation rates should show a pure $q^{3}$ dependence as observed for the star polymers. The slow internal dynamics of the dendrimers might be due to the extremely high polymer densities of about 70 vol \% to 90 vol \% in the rather extended core region. For star polymers computer simulations have shown that the extension of the shell region compared to the overall star dimension is much larger and contains relatively low polymer concentrations $(\leqslant 20 \%)$. $^{7,50}$

As in the case of the star polymers, a calculation of the first cumulant from the measured form factor using Eqs. (5), (7), and (8) would predict a minimum of the first cumulant $\left(\Omega / q^{3}\right.$ plotted versus $\left.q\right)$ close to the location of the form factor peak in the generalized Kratky representation. A power-law dependence $q^{-P}$ of the form factor with $P>5 / 3$ in the intermediate $q$ region would alway lead to such a minimum. We have to keep in mind that the phenomenon of de Gennes narrowing originally denotes a collective phenomenon due to collective movements of many particles. In the intermediate $q$ region of the dendrimer form factor we are not sensitive to the correlation between different blobs. In contrast to star polymers, the dendrimers investigated here are surface fractals rather than mass fractals. Therefore, it is not surprising that there is no direct relation between the form factor and the dynamics of the dendrimers by the formalism of dynamic random phase approximation.

\section{CONCLUSION}

We investigated the structure and the (internal) dendrimer dynamics by means of small-angle x-ray scattering and neutron spin-echo spectroscopy under good solvent conditions.

For the first time we formulated a model that describes the single-particle scattering stemming from the overall compact shape as well as the contribution from the internal density fluctuations for all generations consistently. The hybrid character of dendrimers between colloidal entities on one hand and their polymeric structure on the other hand is accentuated. In addition, we obtained the pair distance distribution functions and the segment density profiles from a model-independent approach via inverse Fourier transformation (IFT) and square-root deconvolution (SQDEC) methods.

Both the model fit as well as the model-independent evaluation of our data reveal that the density profile of all dendrimers $(g \geqslant 3)$ decays monotonically from the center to the outside. The most consistent description of the data has been achieved with the fuzzy edge model. The results for the pair distance distribution function and the density profile are in excellent agreement with those determined via IFT and SQDEC. Down to $g=3$, the shape of $p(r)$ is rather symmetric like those of homogeneous spheres; the shift of the peak position to smaller values of $r / R_{g}$ is fully taken into account by the increasing fuzziness of the surface region. Even though the smearing of the density profile in the shell region increases with decreasing generation number, the steep decay of the form factor in the intermediate $q$ region indicates that even for the lower-generation dendrimers down to $g=3$, the dendrimers are surface fractals rather than mass fractals. So, if a comparison is drawn between star polymers and low- 
generation dendrimers it must be concluded that they are more similar to high-functionality stars. The number of terminal units in the $g=3$ dendrimer is equal to 32. Stars of comparable number of arms have been referred to as "polymeric colloids". ${ }^{50-52}$ We have shown that the vanishing of the higher-order maxima in the sphere form factor is not a sensitive measure for potential structural changes. The changes in the high- $q$ region are, to a large extent, due to the fact that the relative contribution of the blob scattering to the overall scattering $\propto N_{t}^{-1}$ drastically increases with decreasing generation. Between the fifth and sixth generation we observe a major change in the characteristics of the density profile. The extension of the shell region compared to the overall dimension of the dendrimer decreases abruptly, whereas the polymer concentration in the core increases. This is most probably due to the increased tendency of the terminal units to fold back into the dendrimer interior with increasing generation number, as predicted by a number of computer simulations. ${ }^{2,3,7}$ From their molecular dynamics study Murat and Grest ${ }^{2}$ predicted the occurrence of such a transition between the fifth and sixth generation, which is in agreement with our results.

The static results are consistent with our observations on the dynamic properties of the PAMAM dendrimers. Throughout the whole $q$ range of the experiment the dynamic structure factor shows a single exponential decay for all dendrimer generations $g \geqslant 5$, even though the length scales probed at higher $q$ values are much smaller than the overall dendrimer dimension. As opposed to the highfunctionality star polymers investigated by Richter et al. ${ }^{18-20}$ the internal dynamics of the dendrimers is much slower than the center-of-mass diffusion, although the dendrimer dimension and their diffusions coefficients are of the same order as those of the star polymers. Very high segment densities in combination with sterical crowding might lead to very long relaxation rates for the internal dynamics. For all dendrimers investigated here, the diffusion coefficients determined with NSE are close to the values calculated from the concentration-corrected Stokes-Einstein relation using the sphere radii $R_{s}$ determined from the SAXS spectra. Dynamically the dendrimer behavior is close to that of "hard", solid spheres.

${ }^{1}$ D. Tomalia, H. Baker, J. Dewald, M. Hall, G. Kallos, S. Martin, J. Roeck, J. Ryder, and P. Smith, Polym. J. (Tokyo) 17, 117 (1985).

${ }^{2}$ M. Murat and G. Grest, Macromolecules 29, 1278 (1996).

${ }^{3}$ M. Mansfield and L. Klushin, Macromolecules 26, 4262 (1993).

${ }^{4}$ M. Mansfield, Macromolecules 33, 8043 (2000).

${ }^{5}$ T. Prosa, B. Bauer, E. Amis, D. Tomalia, and R. Scherrenberg, J. Polym. Sci., Part B: Polym. Phys. 35, 2913 (1997).

${ }^{6}$ R. Scherrenberg, B. Coussens, P. van Vliet, G. Edouard, J. Brackman, and E. de Brabander, Macromolecules 31, 456 (1998).

${ }^{7}$ R. Lescanec and M. Muthukumar, Macromolecules 23, 2280 (1990).

${ }^{8}$ D. Boris and M. Rubinstein, Macromolecules 29, 7251 (1996).

${ }^{9}$ Z. Chen and S. Cui, Macromolecules 29, 7943 (1996).

${ }^{10}$ P. de Gennes and H. Hervet, J. Phys. (France) Lett. 44, L351 (1983).

${ }^{11}$ A. Topp, B. Bauer, J. Klimash, R. Spindler, D. Tomalia, and E. Amis, Macromolecules 32, 7226 (1999).
${ }^{12}$ A. Lyulin, G. Davies, and D. Adolf, Macromolecules 33, 3294 (2000).

${ }^{13}$ A. Lyulin, G. Davies, and D. Adolf, Macromolecules 33, 6899 (2000).

${ }^{14}$ M. Ballauff, Top. Curr. Chem. 212, 177 (2001).

${ }^{15}$ A. Pötschke, M. Ballauff, P. Lindner, and F. Vögtle, J. Appl. Crystallogr. 33, 605 (2000).

${ }^{16}$ T. Prosa, B. Bauer, and E. Amis, Macromolecules 34, 4897 (2001).

${ }^{17}$ E. Wallace, D. Buzza, and D. Read, Macromolecules 34, 7140 (2001).

${ }^{18}$ D. Richter, B. Stühn, B. Ewen, and D. Nerger, Phys. Rev. Lett. 58, 2462 (1987).

${ }^{19}$ D. Richter, B. Farago, L. Fetters, J. Huang, and B. Ewen, Macromolecules 23, 1845 (1990)

${ }^{20}$ B. Ewen and D. Richter, "Neutron Spin Echo Investigations on the Segmental Dynamics of Polymers in Melts, Networks and Solutions," in Adv. Polym. Sci., Neutron Spin Echo Spectroscopy-Viscoelasticity-Rheology (Springer, Berlin, Heidelberg, New York, 1997), Vol. 134, p. 1.

${ }^{21}$ R. L. Ferla, J. Chem. Phys. 106, 688 (1997).

${ }^{22}$ F. Ganazzoli, R. L. Ferla, and G. Raffaini, Macromolecules 34, 4222 (2001).

${ }^{23}$ O. Glatter, J. Appl. Crystallogr. 10, 415 (1977).

${ }^{24} \mathrm{~A}$. Akcasu, Dynamic scattering from multicomponent polymer mixtures in solution and in bulk, in Dynamic Light Scattering-The Method and Some Applications, edited by W. Brown (Clarendon, Oxford, 1993), p. 1.

${ }^{25}$ W. Burchard, K. Kajiwara, and D. Nerger, J. Polym. Sci., Polym. Phys. Ed. 20, 157 (1982).

${ }^{26}$ A. Akcasu, M. Benmouna, and C. Han, Polymer 21, 866 (1980).

${ }^{27}$ W. Burchard, M. Schmidt, and M. Stockmeyer, Macromolecules 13, 580 (1980).

${ }^{28}$ M. Doi and S. Edwards, The Theory of Polymer Dynamics (Clarendon, Oxford, 1986)

${ }^{29}$ J. Pedersen, J. Appl. Crystallogr. 25, 129 (1992).

${ }^{30}$ J. Pedersen and P. Schurtenberger, J. Appl. Crystallogr. 29, 646 (1996).

${ }^{31}$ K. Müller and O. Glatter, Makromol. Chem. 183, 465 (1982).

${ }^{32}$ S. Goldman, Information Theory (Constable, London, 1953).

${ }^{33}$ O. Glatter, J. Appl. Crystallogr. 14, 101 (1981).

${ }^{34}$ O. Glatter, J. Appl. Crystallogr. 17, 435 (1984).

${ }^{35}$ O. Glatter, J. Appl. Crystallogr. 21, 886 (1988).

${ }^{36}$ M. Daoud and J. Cotton, J. Phys. (France) 43, 531 (1982).

${ }^{37}$ W. Dozier, J. Huang, and L. Fetters, Macromolecules 24, 2810 (1991).

${ }^{38}$ L. Willner, A. Poppe, J. Allgaier, M. Monkenbusch, P. Lindner, and D. Richter, Europhys. Lett. 51, 628 (2000).

${ }^{39}$ G. Beaucage, J. Appl. Crystallogr. 28, 717 (1995).

${ }^{40}$ L. Willner, O. Jucknischke, D. Richter et al., Macromolecules 27, 3821 (1994).

${ }^{41}$ M. Adam and D. Lairez, Fractals 1, 149 (1993).

${ }^{42}$ A. Naylor and W. Goddard, J. Am. Chem. Soc. 111, 2339 (1989).

${ }^{43}$ J. Pedersen, Adv. Colloid Interface Sci. 70, 171 (1997).

${ }^{44}$ B. Hammouda, J. Polym. Sci., Part B: Polym. Phys. 30, 1387 (1992).

${ }^{45}$ D. Tomalia, A. Naylor, and W. Goddard, Angew. Chem. Int. Ed. Engl. 29, 138 (1990).

${ }^{46}$ M. Monkenbusch, R. Schätzler, and D. Richter, Nucl. Instrum. Methods Phys. Res. A 399, 301 (1997)

${ }^{47}$ S. Rathgeber, L. Willner, D. Richter, A. Brulet, B. Farago, M. Appel, and M. Fleischer, J. Chem. Phys. 110, 10171 (1999).

${ }^{48}$ Since bulk densities for PAMAM dendrimers with $g \geqslant 6$ are not available, we used the density $\rho=(1.22 \pm 0.122) \mathrm{g} \mathrm{cm}^{-3}$ of the fifth generation for all dendrimers (taken from Ref. 53).

${ }^{49} \mathrm{~J}$. Dhont, An Introduction to the Dynamics of Colloids (Elsevier, Amsterdam, 1996).

${ }^{50}$ G. Grest, K. Kremer, and T. Witten, Macromolecules 20, 1376 (1987).

${ }^{51}$ J. Stellbrink, J. Allgaier, M. Monkenbusch, D. Richter, A. Lang, C. Likos, M. Watzlawek, H. Löwen, G. Ehlers, and P. Schleger, Prog. Colloid Polym. Sci. 115, 88 (2000).

${ }^{52}$ C. Likos, H. Löwen, M. Watzlawek, B. Abbas, O. Jucknischke, J. Allgaier, and D. Richter, Phys. Rev. Lett. 80, 4450 (1998).

${ }^{53}$ S. Uppuluri, D. Tomalia, and P. Dvornic, ACS PMSE preprints 77, 87 (1997).

${ }^{54}$ For simplicity we denote with $I_{i}(q)(i=\exp$, IFT) the absolute scattering cross section $d \Sigma / d \Omega(q)$. 